\title{
A fine-grained multi-source measurement platform correlating routing transitions with packet losses
}

\author{
Pascal Merindol $^{*, a}$, Pierre David $^{\mathrm{a}}$, Jean-Jacques Pansiot ${ }^{\mathrm{a}},{\text { Francois } \text { Clad }^{\mathrm{b}} \text {, Stefano Vissicchio }}^{\mathrm{c}}$ \\ a Universite de Strasbourg, France \\ b Cisco Systems, USA \\ ${ }^{\mathrm{c}}$ University College London, UK
}

\section{A R T I C L E I N F O}

\section{Keywords:}

Active and passive measurements

IP routing convergence

Control plane

Date plane

Management plane

\begin{abstract}
A B S T R A C T
In this paper, we are interested in the relationship between packet losses and routing changes in an operational network. To do so we designed and deployed DCART, a monitoring platform over RENATER, the French research and education network. Our platform collects four data sources using both active and passive measurements in order to unveil their temporal correlations. Active probing allows especially for measuring packet losses on specifically crafted data flows. Those flows explore several load balanced paths and ease the revelation of forwarding loops. Passive monitoring is achieved by listening to all routing updates from IS-IS, the intra-domain routing protocol in use, and by retrieving tickets generated by the Network Operations Center (NOC).

During our monitoring campaign, we observe that most of the series of loss were correlated to routing events either because routing changes lead to inconsistent state transitions, or because faulty - and so lossy - links trigger numerous periods of link flapping. In particular, we show that losses due to forwarding loops resulting from inconsistent routing states are quite common when links come back after an outage. We also show that link flapping sometimes induce very long lasting lossy periods frequently unnoticed by the NOC. A lightweight monitoring platform such as DCART could be used to better anticipate recurrent network outages and to improve the ticketing system.
\end{abstract}

\section{Introduction}

Internet service providers (ISP) seek a very high degree of reliability for their network, the $99.999 \%$ (so called five nines) availability ratio is often mentioned. This demand is triggered both by applications requiring strong network quality such as voice calls, and by clients asking for stringent Service Level Agreements (SLA) that require to be verified on the ground.

To achieve this goal, it is not only necessary to monitor the network from different points of views (e.g. at the control, data and management planes), but also to correlate these informations. Understanding the nature of the correlations between them and looking for dependencies is a first step towards the general enhancement of any network.

In this paper, we present many experimental results and related findings obtained thanks to a new kind of monitoring infrastructure whose first prototype is called DCART. DCART is a distributed platform running over an operational network (RENATER, the French research \& education network); it gathers data from several sources. Constant rate data probes monitor the data plane, recording losses, ICMP error messages, path changes and one-way delay variations. Carefully crafted one-way probes allow an ISP to detect and locate forwarding loops and to monitor different ECMP ${ }^{1}$ paths. An Interior Gateway Protocol (IGP) listener, in our case IS-IS [1], collects all changes in the intra-domain routing control plane. The management plane is tracked through tickets issued by the Network Operations Center (NOC). A design goal of this platform is to be as independent as possible from the network infrastructure, so data probers as well as the IGP listener are implemented on dedicated devices, using only open source software and commodity hardware.

As our objective is to study the relationship between packet losses and routing changes, DCART allows fine correlations between those various sources. In this paper, we are trying to answer questions such as: what is the severity and the nature of packet loss during a routing transition? Is a link up producing losses? Are link down or rather up

\footnotetext{
* Corresponding author.

E-mail addresses: merindol@unistra.fr (P. Merindol), pda@unistra.fr (P. David), pansiot@unistra.fr (J.-J. Pansiot), fclad@cisco.com (F. Clad), s.vissicchio@ucl.ac.uk (S. Vissicchio).

${ }^{1}$ Equal-Cost Multi-Path.
} 


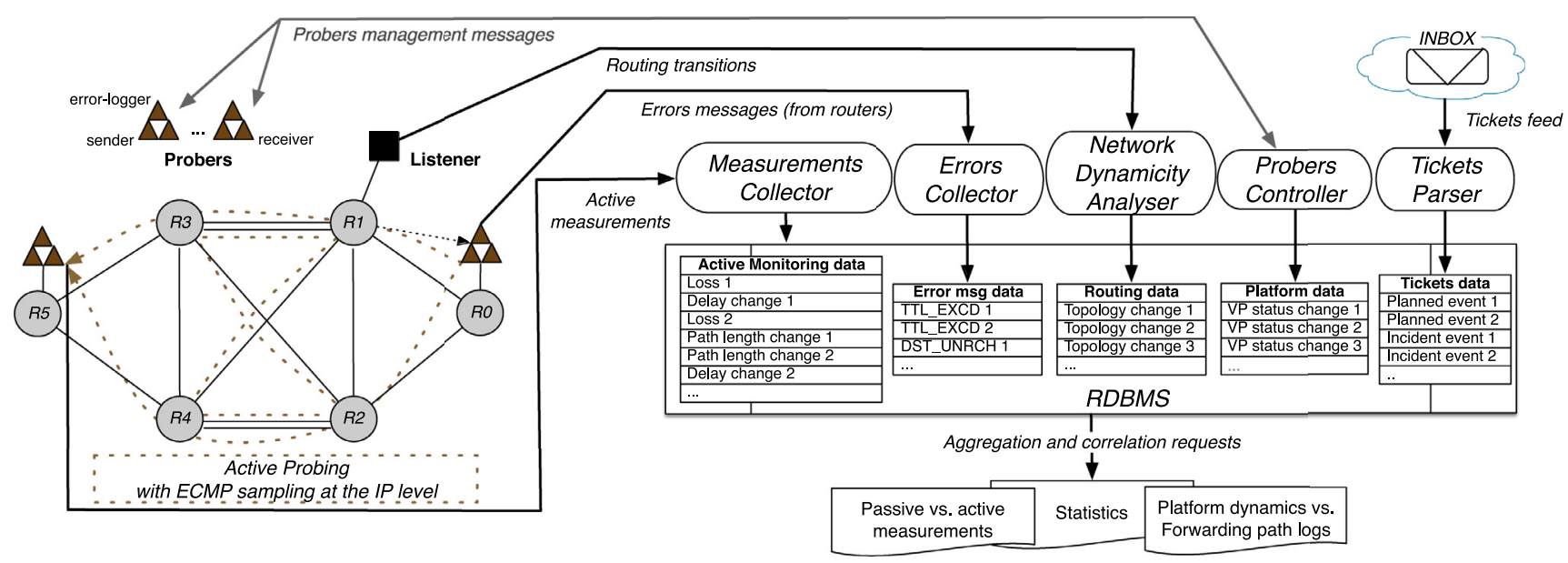

Fig. 1. The architectural overview of DCART. (For interpretation of the references to color in this figure legend, the reader is referred to the web version of this article.)

events resulting in forwarding loops? What is the share of packet loss not triggered by routing changes? What are their root causes? What is the relative impact of routing changes due to failures compared to planned maintenance? Is the impact of network outages identical across all flows between the same end-points? Generally speaking, we try to determine the origins of data plane outages to understand wether and how an ISP can mitigate them.

In order to answer this type of questions, DCART collects all these sources of information and store them together with a timestamp (processes are synchronized using NTP) in a relational database. Thereafter, these time series can be correlated through these timestamps by suitable queries to the database. Key strengths of our experiment may be summarized in three points. First, we gather almost one year and a half of passive data and more than four months of active data on a real ISP. Second, we propose and use several methods for aggregating, correlating and analyzing the relations between distinct sources of events. Then, we present many networking results using different perspectives such as spatiotemporal graphs that ease the data visualization.

Among the various measurement results we collect and present in this paper, we observe three most notable results:

(i) although not numerous, transient forwarding loops are not negligible as they induce loss sequences lasting up to several seconds. Their prevalence is higher at link up than at link down.

(ii) long periods of link state flapping are correlated with severe error rates. Most losses appear in long series of short bursts as long as the faulty link is active and disappear when it goes down.

(iii) most down events, including those related to faulty links, trigger significant blackhole periods. Such periods appear when no active outgoing interface is available so that it results in long series of consecutive loss defined as loss sequences. Most of them are not related to any forwarding loop indication.

The purpose of DCART is to identify the main recurring troubles occurring in IP operational networks. Our platform does not aim at mitigating such problems on its own but rather to help network administrators to take most pertinent actions related to them (e.g., shut down a flapping link and starting its maintenance as soon as possible). After a short description of the DCART architecture in Section 2, we describe our measurement campaign over the RENATER network in Section 3. Section 4 provides a first analysis of data obtained from each data source independently, while Section 5 is devoted to their correlation. Most of temporal correlations between routing events, loss sequences and loops can be performed at a fine time resolution, typically on the order of $100 \mathrm{~ms}$. Section 6 delves into specific events, illustrating the power of crossing multiple data sources in order to understand non trivial correlations. For example, we show how a link may start to become lossy long before anything is detected at the routing plane. We also show how series of short loops on distinct links may impact a given data flow for a while during a network transition.

\section{DCART architecture}

In this section, we describe the design and the features of our measurement platform called DCART that stands for "Dynamic reConfiguration Analysis with Routing Traces".

\subsection{Design objectives}

DCART collects informations from multiple data sources in order to analyze the causes and the effects of the dynamicity of a real ISP network infrastructure. The main feature of DCART is its ability to perform fine grain temporal correlations between these distinct sources of informations. More specifically, our objectives in designing the architecture of DCART are:

- Monitoring simultaneously the data, control and management planes. Our aim is to provide as much informations as possible while keeping a limited impact on the network under scrutiny;

- Allowing and performing correlations between distinct measurement sources to provide many insights into network dynamics;

- Designing specific one way active probes: we aim to uncover generally neglected details about the network data plane, such as forwarding loop occurrences and their location at the data flow level;

- Gather data for later analysis: since this is an exploratory work, data must be kept in a database with a powerful querying facility. We choose a relational database with the SQL language;

- Be vendor independent, scalable and flexible: DCART does not rely on specific hardware or software characteristics, whether for the ISP network devices or for internal DCART components. Probers are low cost commodity hardware and new components can be easily added to increase coverage, accuracy or reliability.

\subsection{Architectural overview}

Fig. 1 describes the main components of the DCART architecture. The first high level component consists in a set of active probers. They are distributed on the monitored network and are made of three subcomponents: the sender, the receiver and the error-logger. The sender 
emits ICMP datagrams to all other probers in order to form an active probing full mesh at the global scale. A receiver records ICMP datagrams emitted from a given sender of another prober while the errorlogger sub-component records ICMP error messages sent from the intermediate routers. Probers are then able to detect packet losses for multiple data flows independently as well as other informations such as the variations in terms of delay and number of hops of one way loadbalanced paths. In addition error-loggers allow DCART to obtain information on forwarding loops.

The second component is the routing listener. It passively collects IGP routing messages. These informations provide a comprehensive knowledge of each logical signalization event as well as the ability to understand the topology, its evolution including details on all load balanced forwarding paths at a given time.

The third component parses and records operational tickets issued by the NOC: this provides valuable information supplied by network engineers to validate some hypothesis or understand the root cause of detected problems.

Collected informations from all these sources are stored in a relational database in order to perform a-posteriori analysis, statistics and correlations. The remainder of this section provides details on each individual component.

\subsection{Active probers: monitor the data plane}

Active measurements are performed by probers located at carefully selected points on the network in order to maximize the coverage of monitored links. They are directly connected to the routers or L2 devices within the ISP and synchronized using the Network Time Protocol (NTP).

Each sender periodically emits ICMP Hello Request datagrams to its associated receivers: this < source, destination $>$ couple forms a one way flow. We use ICMP rather than UDP or TCP since we observed that, at least on RENATER, some routers do not send back error messages when receiving UDP or TCP packets while they always return such messages when receiving ICMP probes. The sender inserts a timestamp and a sequence number in each probe packet sent. This way, the receiver is able to handle the probe packet on its own: nothing is recorded at the sender side and no other information exchange is required between the two endpoints. A receiver only logs what it interprets as an anomaly at the flow granularity, e.g. out of order packets. To minimize the churn of our measurements on the user traffic, replies are never sent to the sender.

To provide additional information about the occurrence of a forwarding loop, the error-logger records ICMP "TTL Exceeded" messages which are sent back by intermediate routers when a probing datagram is in fault, i.e. when its TTL is strictly lower than 1 . To force the detection of such network anomalies, we configure senders to use a value just slightly higher than the network diameter as the initial TTL of probes. This way, we both ensure that collected ICMP "TTL Exceeded" messages are actually loop indications and, mostly, minimize the likelihood to miss short-lived loops. More precisely, for subsequent messages, we alternate the initial TTL to two consecutive values. This guarantees that we are also able to unambiguously infer the actual location of any transient loop. Indeed, only two-node loops can occur if all link weights are symmetric [2] as it is the case in RENATER so that it is easy to show that two consecutive TTL initial values will trigger error messages from the two routers involved in the loop.

ISPs often provide fault-tolerance and load-balancing features through ECMP, which are generally implemented on routers using a hash function involving several packet header fields including the IP destination address. Indeed, per-flow or per-destination load balancing avoid misordering and performance issues at the TCP level. For some $<$ source, destination $>$ pairs, packets are thus sent on one path or another depending on specific header fields. Different ECMP paths may have very different data plane performance, even for the same source- destination pair [3]. As some routers use a hash function based only on the IP header, we make use of several IP addresses per prober. If our probers are provided with a $/ n$ subnet prefix, we can use $2^{32-n}-3$ addresses. For example, given the use of the 198.51.100.0/29 prefix for the connection of a given prober, its gateway router will use the IP 198.51.100.6, and the range 198.51.100.1-5 will be available for the prober. In our deployment (see next section), each sender cyclically emits packets to all destination IP addresses of a given receiver. Packets towards the same destination address form a flow. Note that we could also have used several IP source addresses per prober in order to explore more ECMP paths, however we did not consider this option to keep source addresses for other operations such as management. Receivers and error-loggers send the collected information, e.g. packet losses and recorded forwarding loops of each flow, to a central measurement collector. Log files are sent with $r$ sync $^{2}$ using an option to limit bandwidth usage in order to reduce the impact of sending large files.

Probers are centrally monitored by a controller which periodically checks their states in order to automatically refine the probing at the flow granularity. That is, when the controller detects the failure of a prober $p$, it asks other probers to turn off their useless active subcomponents related to $p$. When the controller observes that $p$ comes up it notifies other probers to relaunch their related sub-components. In case of software crash, note that probers also periodically try to restart their own crashed subcomponents as long as the controller does not explicitly notify probers to turn them off.

Probers are built upon commodity hardware, i.e. Raspberry Pi, using open-source software only. This allows large-scale deployments at a very low cost. However, we experienced hardware limitations, mostly concerning CPU and network interface, as well as flash-disk failures.

\subsection{IS-IS listener: monitor the control plane}

Active measurements are complemented with the passive listening of messages carried by the link-state IGP in use, IS-IS in our implementation. Upon each local change on its state, a router floods a LSP (Link State PDU, the elementary topological message of IS-IS routers) to all other participating routers. We built a program based upon an opensource routing daemon [4] which listens to IS-IS messages, parses and dumps them in the standard MRT format [5]. This program runs on a Linux server directly connected to an ISP router. Furthermore, several extensions have been designed to build and manipulate all distinct transient topologies resulting from routing changes. Those network states are associated to the set of time intervals during which the network has undergone the corresponding routing modification. Such time-based topologies enables spatiotemporal correlations that require to check the forwarding path in use at a given moment.

\subsection{ISP tickets: monitor the management plane}

For most ISPs, a NOC sends informational tickets to its clients or partners to report maintenance periods or network outages. Their content is supplied by network engineers and provide human-readable informations on the reported event. We developed a ticket parser specific to our deployment network. It both classifies reported events into a type (incident, maintenance) and a sub-type (e.g. client, router, link) and extracts spatial and temporal informations available from the original tickets.

\subsection{Database: perform statistical analysis}

Raw data resulting from the previous sources are a-posteriori processed and stored in a relational database (PostgreSQL). Using such a

\footnotetext{
${ }^{2}$ https://rsync.samba.org.
} 


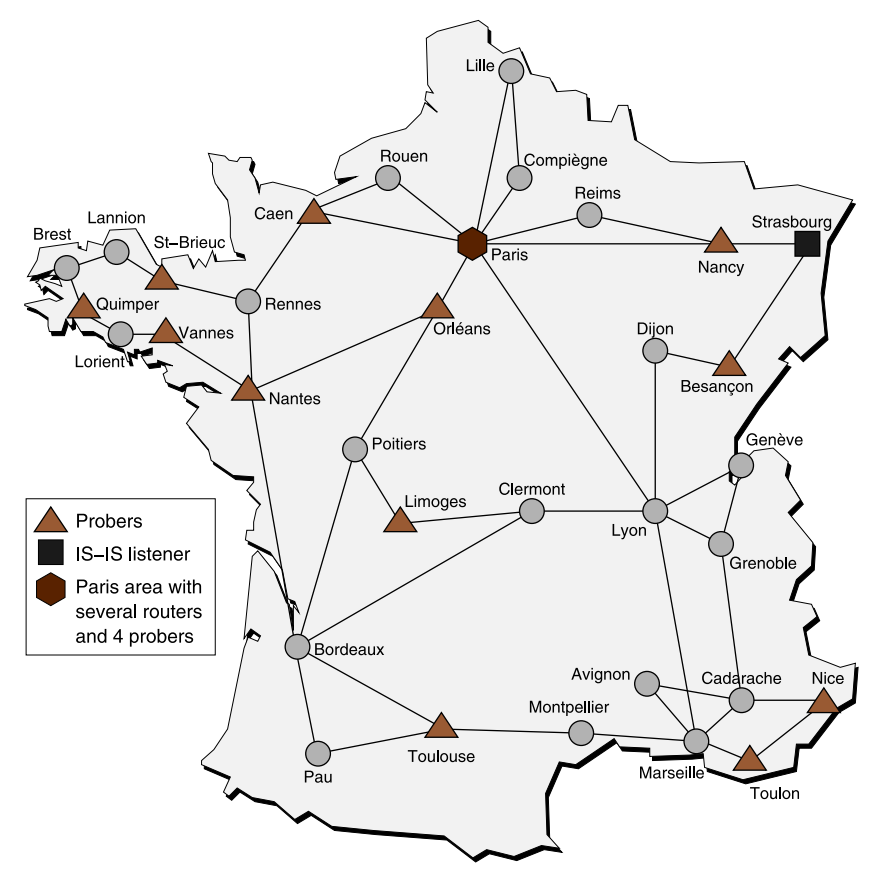

Fig. 2. Simplified map of the DCART deployment on RENATER. (For interpretation of the references to color in this figure legend, the reader is referred to the web version of this article.)

relational database provides a structured view with integrity constraints which helps to strengthen data and their relationships. The SQL language allows both easy querying and good request performances.

All analysis described in this paper has been performed on refined data stored in the relational database after the measurement campaign has been completed. A future work would be to incrementally process data and perform analysis in real-time in order to detect network glitches long before they become problems.

\section{The measurement campaign}

\subsection{RENATER, the network under scrutiny}

RENATER, the French National Research \& Education Network depicted in Fig. 2 is a transit network made of 79 routers located in all the largest French cities, connected by 234 IP links, including parallel physical links. The routing IGP in use is IS-IS, all routers are level 1 routers in the same area. Most routers enable the ECMP feature to load balance the traffic across several links. However, some of them only apply the hashing function to the IP header (e.g. Paris and Lyon's Cisco CRS routers deploying IOS XR). In addition to the IP routing protocol, RENATER uses MPLS switching along tunnels built with LDP $^{3}$ The DCART traffic is tunneled through LDP tunnels following standard IGP paths. Among other information useful to attach loops to loss sequences, we capture the MPLS header quoted in "TTL Exceeded" error messages. Indeed, error messages resulting from forwarding loops contain a quotation of the original faulty message in transit. We verify that our probes are tagged with MPLS labels. Most RENATER routers send many "TTL Exceeded" error replies, thanks to a loose rate limiter, while we did not receive any "Destination Unreachable" reply during our campaign.

\subsection{About the campaign and its calibration}

We now describe our real-world scenario implementation to emphasize the fine-tuning and the granularity of DCART. To build the DCART platform, we have been granted the possibility to deploy monitoring nodes, our set of probers, in the operational network. We deploy 16 probers directly connected to routers located on several french cities. Fig. 2 shows a rough map of RENATER in which we summarized some information for readability. Notably, Paris sub-network is represented as a single (hexagonal) node but includes about 20 routers and 4 probers.

The location of probers result from two considerations: the operational deployability cost and the link coverage optimization. We use a greedy heuristic where, at each iteration, we look for the sender location able to theoretically cover the most forwarding loops among any possible feasible locations. For each candidate, we compute the total number of not yet covered potential loops from which it can collect evidences: this is achieved by considering all cycles in the graphs resulting from the merging of all outgoing paths before and after the failure of any component. With the resulting configuration, DCART has the ability to discover most loops and covers more than $80 \%$ of the links used in normal operational mode, i.e. when no failures occur. Our probers placement enables us to send probing packets over most active network paths. DCART monitors almost all links and routers except unused backup links that cannot be monitored when there is no failure and leaf parts which do not have an attached DCART prober.

DCART is able to collect routing states and forwarding phenomenons related to ECMP load balanced paths. However, for practical reasons, our deployment does not monitor all these paths. The major limitation of the adopted hardware is that it does not support a high probing rate. Namely, we experimentally verified that sending probes to 15 destinations every $20 \mathrm{~ms}$ saturates a R-PI 2 CPU. ${ }^{4}$ Indeed, in such a case, our probers only send (and thus receive) approximatively $50 \mathrm{~KB}$ / $\mathrm{s}$ each (probe packets are the smallest possible, i.e., 64 bytes), while the capacity of the Ethernet interface of a Raspberry Pi should theoretically support $100 \mathrm{Mb} / \mathrm{s}$ and the typical link capacity of RENATER is greater than $10 \mathrm{~Gb} / \mathrm{s}$. If the traffic load is controlled thanks to small packets, their processing is very time consuming for the CPU of the Raspberry $\mathrm{Pi}$ that clearly exhibits an overload in such a situation (i.e. it is totally saturated in practice). One can easily overcome this limitation by carefully deciding which prober monitors which paths at which frequency. Exploiting the high coverage of our probers enables us to continuously monitor most transit links at a high probing rate while using a low rate at leaf links. Precisely, active measurements are performed by default every $40 \mathrm{~ms}$ between every pair of probers. As a result, each monitored pair from source $a$ to destination $b$ is probed every $40 \times k \mathrm{~ms}$, where $k$ is the number of flows used between $a$ and $b$. A flow consists in a single forwarding path from $a$ to $b$. In practice, we use $k=5$ such that if several ECMP paths exist between a given pair of probers, we have almost $94 \%$ of chances to explore distinct physical paths with our 5 flows: $\left(1-\frac{1}{2^{4}}\right) \times 100=93.75$. On RENATER, our listener ECMP feature shows that some specific degraded topological situations may lead up to 18,000 distinct paths for a single pair of probers. For most pairs of probers, paths are generally longer if the network state is degraded, increasing so the path diversity capabilities of ECMP. It is thus very difficult to explore a large share of this existing potential in any cases. Our 5 flows are rather a way to actively confirm the presence of some path diversity and so, in some favorable cases, measure new indicators (e.g. a loss sequence that occurs on another path) that we may miss otherwise with $k=1$. On the whole listener collecting period, DCART records more than 4000 distinct global

\footnotetext{
${ }^{4}$ Central Processing Unit of Raspberry Pi 2. New generations of Raspberry Pi hardware are more powerful and are likely to support higher probing rates.
}

\footnotetext{
${ }^{3}$ Label Distribution Protocol: the simplest protocol used to distribute MPLS labels.
} 
network states, to compare with almost 70,000 global state transitions. Most states are degraded states resulting from - sometimes multiple failures occurring on several long term standard topologies.

Logs are temporarily stored on probers ${ }^{5}$ and periodically sent to the database via the RENATER network itself. Thanks to the high redundancy of RENATER, data collection was not impacted during transit link failures. We also engineer logs to improve scalability. To reduce measurement overhead and limit the load on probers, the monitoring controller fine-tunes the events that are logged by each prober. In our ongoing measurement campaign, we focus on routing events, such as topological or configuration changes, and their impact on data plane traffic. Receivers record incoming packet information only in the case of significant changes in key performance indicators, i.e. when the oneway-delay or the number of hops vary upon a given margin regarding the previous recorded values. Such thresholds are verified both with respect to either the previous measurement for the same flow or the previous one in other flows. For example, receivers do not log consecutive and subsequent measurements for which the delay variation is less than $1 \mathrm{~ms}$. However, they log all possible raw information (i.e. the packet arrival time, its sequence number, its delays and the number of hops it traverses) since then the flow is impacted by any significant changes or packet ordering anomaly (loss or out-of-order delivery). Finally, we log probe arrival data only at the receiver side, inserting timestamps in the ICMP payload to estimate one-way delay. Such finetuning globally reduces the size of a 1 hour active measurement logs from $1 \mathrm{~GB}$ using raw reporting to $72 \mathrm{MB}$. A heartbeat mechanism at a rate of 2 packets per second is used to track probers status and accounts for a large part of this data.

Table 1 provides major quantitative and temporal raw informations about our measurement campaign. While we collected listener events and ISP tickets for about 16 months, we stop collecting data from our probers after less than 4 months, due to a weakness in their SD cards. Thus, correlations between passive and active data is limited to 4 months. Finally, note that timestamps for both active and listener events are expressed as microsecond numbers. Although their accuracy is certainly closer to the millisecond scale for multiple reasons - and synchronization limitations in particular, DCART does not require such an accuracy. It can operate at the order of a tenth of a second.

\section{Single source analysis}

This section describes the main results obtained from each data source separately. We start with the listener that reveals numerous cases of more or less severe link flapping.

Events reported by each source frequently occur in bursts resulting from a recurring cause such as an unstable link. In order to aggregate related raw events, we define temporal and spatial thresholds under which raw events are grouped into higher level events.

\subsection{Listener: link flapping is the rule}

The listener is certainly the most efficient component of our infrastructure as its deployment and maintenance costs are almost negligible regarding the benefits it offers. We are able to follow the entire knowledge of the network routing dynamics, i.e. the control plane information. The listener captures and filters all link state changes in order to classify them according to their high level nature. Since the RENATER network has only one IS-IS area, we do not need to merge information from several routers. Moreover, lost LSPs can be easily detected because they are numbered. Such a loss could be due to a listener error, a transmission error between routers or a partition of the network. Hopefully, it appears that lost LSPs are very rare and most of

\footnotetext{
${ }^{5}$ The main weakness of the Raspberry hardware seems to be the SD card that fails when too much logs are written in a row.
}

Table 1

Details of our measurement campaign: collecting periods and number of events.

\begin{tabular}{ll}
\hline $\begin{array}{l}\text { Active probing } \\
\text { Logged losses }\end{array}$ & \\
Error messages & 529,287 \\
Collecting interval & {$[2015-03-23,2015-07-14]$} \\
Logged ICMP packets & 4137 \\
IS-IS listener & \\
Collecting interval & {$[2015-03-23,2016-07-29]$} \\
Topological LSP processed & 69,227 \\
Active days & 453 \\
NOC tickets & \\
Collecting interval & {$[2015-03-23,2016-07-29]$} \\
Processed messages & 2196 \\
Unique tickets & 893 \\
\hline
\end{tabular}

them occur while a router is starting up after a failure. Both the listener and IS-IS in general are reliable enough to provide consistent informations.

We dig into more than one year of topological changes (453 days), i.e. the ones that imply modifications in the network topology graph, and group them according to their nature and impact (link up or down, or weight change) and their scale (link or router). In Table 2, one can notice that routing changes are frequent in general: several dozens on average per day, and more than 2000 in the worst day. Weight and router wide changes are very rare events while link up/down events are, by far, the most frequent. We also observe a significant gap between the median and the average of link events. It is due to severe periods of link flapping: in practice, we observe that a few periods, that last several days each, account for more than half of routing changes. In the remainder of the paper, we will generally consider both raw events and several forms of temporal aggregations but only for link events. We will avoid to spatially group raw events into wider scale networking events such as bi-directional links, line-cards, routers or even Shared Risk Link Group (SRLG) for several reasons. First, they seem to be rare enough to be considered as almost negligible; second, it is difficult to correctly build such networking component-wide events since the routing protocol does not directly provide such information and they do not suit for precise temporal correlations as they are composed of several timestamps.

However, most of the collected raw events result from flapping issues: a temporal aggregation is unavoidable to understand and correctly handle the actual nature of underlying events. Formally, we define a flapping period (flap) as a time period where each $2 \mathrm{~h}$ time window or less contains at least 3 events for the same directed IP link. We compute all distinct longest periods for each link. Note that a simple outage (a "down" then "up" pair) is not classified as a flap because the four changes occurring on the two directions of the same link account for only two raw events for each direction.

Using this aggregation, we are able to distinguish isolated outages from flaps. An isolated event is de facto an event not included in a flap, and we also consider it as a high level event. The result is impressive: almost $95 \%$ of link state changes fall in the flap category, meaning that the frequency of routing events (isolated or flap period) drops to less than 13 events per day (see Table 3: $8.47+4.32 \approx 13$ ) instead of 69227 / $453 \approx 153$ raw events without any aggregation (see Table 1). As quantified latter in this subsection, we will see that isolated events occur during very short down-up periods. In most tables provided in this section, we provide statistical indicators at the day scale in order to manipulate tangible data. The notion of busy day then follows naturally as a day with at least one event. For example, looking at Table 3, the $74 \%$ of busy days on the isolated events line means that, on average, less than 2 days per week are free of any isolated changes $(7-0.74 \times 7=1.82)$. It is worth noticing that this value of $74 \%$ remains the same considering globally all of routing events.

While isolated events produce 8.47 state changes in average (and so 
Table 2

About topological changes, a spatial aggregation attempt of listener events (per day statistics).

\begin{tabular}{lllll}
\hline Types & Average & Median & Max & Total \\
\hline Link down/up & 37.35 & 6 & 2074 & 16,848 \\
Router down/up & 0.3 & 0 & 8 & 136 \\
Weight change & 0.12 & 0 & 4 & 57 \\
\hline
\end{tabular}

Table 3

Isolated events and flaps, a temporal aggregation of listener events (per day statistics).

\begin{tabular}{lllll}
\hline Event types & Average & Median & Max & Busy days (\%) \\
\hline Isolated events & 8.47 & 5 & 84 & 74 \\
Flap periods & 4.32 & 2 & 44 & 69 \\
\#Raw events in flaps & Average & Median (busy day) & Max \\
& 137 & $16(36)$ & 8070 \\
\hline
\end{tabular}

$8.47 / 0.74 \approx 11.4$ per busy day), flap periods lead to about 200 state changes per busy day $\left(137 \times \frac{1}{0.74}\right)$. Using Table 3, one can accurately deduce that only $8.47 /(137+8.47) \times 100 \approx 6 \%$ of raw events fall in the category of isolated events. As the worst example collected a given IP link, we have observed that during three subsequent days, at least 4000 events have been reported per day to the listener with a maximum peak of 8070 in a single day. This period ranges from 2015-09-09 to 201509-11 on the link connecting two routers in Marseille.

With this first analysis, we can conclude that link flapping is the rule. Put in more networking words, the failure of a link does not look to predominantly result from a physical fail-stop model but rather from intermittent failures due, for example to software problems or degradation of hardware electronics or link quality. Despite the prevalence of flapping periods that sometimes last so long that they cumulate, and so interfere, we did not observe many raw event interferences among independent network locations. More precisely, it is very rare that down/up state transitions of a given device is interlaced with transitions of another device. In practice, we did not found any SRLG events in our study.

Note that numbers presented in Table 3 should be divided by 4 to obtain the actual number of link outages: an IP link has two directions and a down followed by an up transition results in four state changes. Considering this per outage counters, the median of the number of isolated network outages occurring per day is only slightly larger than one. Eventually, one can observe that the intensity of flaps is quite impressive even when considering the median, i.e. they account for 9 network outages per busy day (36/4).

Fig. 3 shows a different perspective that also highlights these first surprising results. ${ }^{6}$

This figure, and other following spatiotemporal illustrations in general, aim to first show the coarse grain big picture that introduces the general analysis before digging statistically into it. In particular, this first spatiotemporal illustration comes with several advantages: it offers a first look at our different collecting periods showing that there are many control events, mostly included in flaps.

For consistency and readability reasons, we distinguish several time periods on the x-axis: the first one, on the left of the black vertical arrow is the one that we will latter cross with active probing (except the light gray period in background where DCART probers were down), while the second, on the right, after week 29 , only offers passive monitoring

\footnotetext{
${ }^{6}$ For all spatiotemporal figures in this paper, we recommend to use an electronic version and to arbitrarily zoom on pieces of interest as most of them are provided in a vector format when possible. However, representing losses, or numerous events in general, sometimes requires to use non conservative formats.
}

informations with the listener and tickets. Statistics about passive measurements are computed on the whole period including the light gray period but not the empty one (from the end of 2015 until end of January 2016) where the listener was down during a bit more than a month. On the y-axis, we sort routers according to their degree and plot all events (both isolated and flapping ones) related to each of their outgoing links. Note that we plot each topological change at the IP link scale.

We can observe several remarkable patterns revealing distinct network outages. An orange horizontal line implies link flapping while a black column reveals a dependent group of links that can range from a line-card up to the more general case of SRLG including router-wide events such as the Paris2a router fail-stop at week 36. An orange column then depicts a flapping at the scale of a group of links, typically a router flapping (as highlighted with the second violet frame at week 37 - also look at Fig. 4(b) for details). Generally speaking, the most visible patterns are long orange twin-lines, such as the ones that range from week 7 to 10 of 2016 between Paris2a and Compiegne or the ones between marseille- 1 and marseille- 2 at week 37 that consist in the most intense flapping of our passive dataset (more than 15,000 events). These patterns illustrate very long periods of link flapping in both directions of a given physical link. In particular, one remarkable event that we are able to correlate with active measurements is recognizable through the two small lines in the left down corner (first violet frame at week 15 - also look at Fig. 4(a) for details). This aggregated event results from a 3 days period of severe link flapping between Paris1 and Lyon1 during the second week of April. Next sections will deeply focus on such a phenomenon and on this one in particular. We also observe many orange square and rectangle patterns that result from relatively short flapping periods of groups of parallel links, look at Vannes or Nantes router timelines near the week 19 of 2015.

Fig. 4 provides details about the shape of two kinds of flaps. We select these two specific time intervals for zooming because they belong to the crossed period between data and control plane and Section 6 provides a detailed short story about the flap event illustrated in Fig. 4(b). Instead of aggregating the listener raw data into orange flaps, we provide here some insights about the internal patterns inherent to a flap. We both plot the nature of the listener raw event (i.e. a green star is an up event, the link comes up, while a red square is a down event) and temporal details about their interlacing and time distribution in general. First, we can observe that, most of the time, up and down events occur within the same minute or even less and that the flap intensity is high, i.e. dozens of state changes can occur in less than one hour. Moreover, it seems that as soon as the link or the router goes down, it comes back just after and then goes down again but generally a while after, i.e. inter-outage durations are longer than the intra-outage ones. In the following sections, we will dig into potential correlations with other sources of data to better understand the possible root causes of such flap characteristics.

We also plot on Fig. 5 the link outage durations, i.e. delays between down and up state changes, and their inter-arrival periods, i.e. delays between up and down state changes. The orange line of Fig. 5(a) provides the cumulative distribution function for outage durations considering only events belonging to flaps. We can see that $90 \%$ of them last less than $2 \mathrm{~min}$. Most outages are not really long, i.e. $99 \%$ of them are shorter than $10 \mathrm{~min}$. Note that on RENATER, there generally exists enough redundancy for most < source, destination > pairs, so link outages are much longer than data outages as we will see in Section 4.3. Fig. 5(b) shows the inter-event time distances on a per component basis. We observe that in about $75 \%$ of the cases, the delay between an up event and the next down event in the same flap period is less than 2 min. However, we note that inter-outage duration is significantly longer than outage duration.

Since isolated events are far less numerous than flap ones, the black line on the same figures shows the distribution with only isolated events: we can see that events are much more spaced out in average 


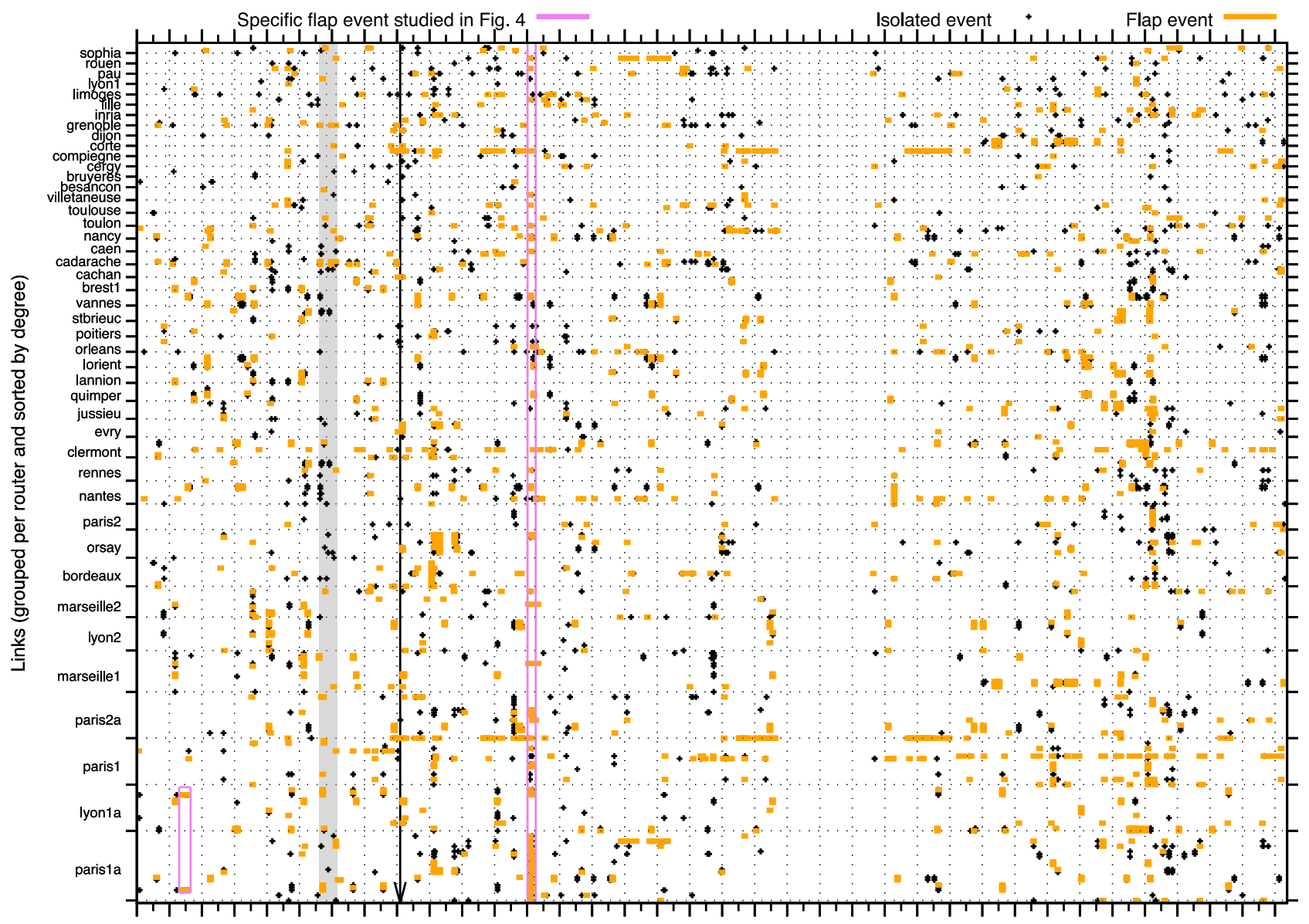

$\begin{array}{lllllllllllllllllllllllllllllllllllllllll}13 & 15 & 17 & 19 & 21 & 23 & 25 & 27 & 29 & 31 & 33 & 35 & 37 & 39 & 41 & 43 & 45 & 47 & 49 & 51 & 53 & 02 & 04 & 06 & 08 & 10 & 12 & 14 & 16 & 18 & 20 & 22 & 24 & 26 & 28 & 30\end{array}$

Time (Week Number 2015-2016)

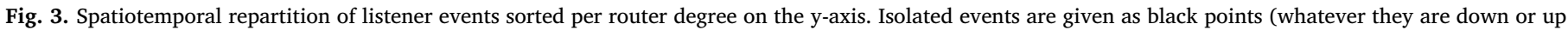

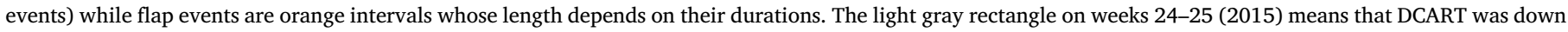

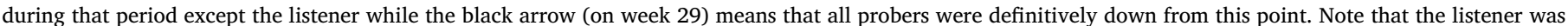

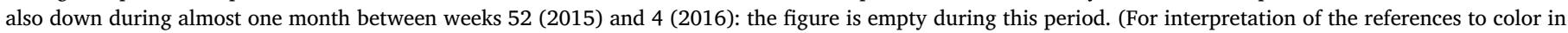
this figure legend, the reader is referred to the web version of this article.)

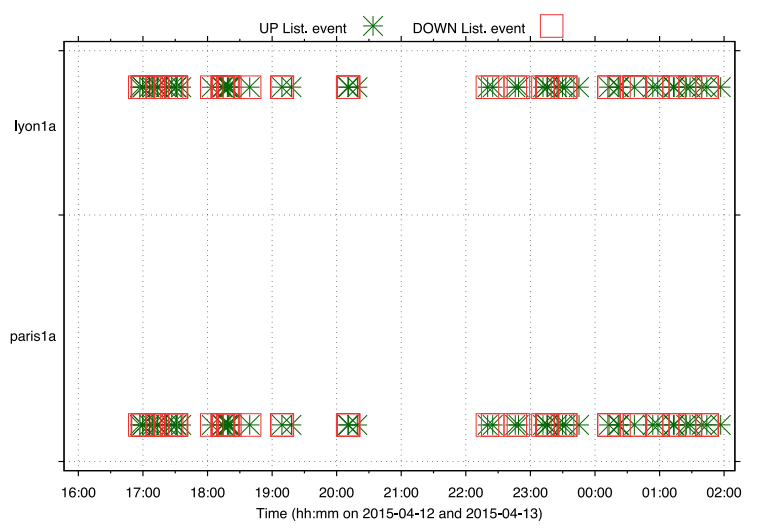

(a) Single link flapping on a ECMP bundle (Paris - Lyon)

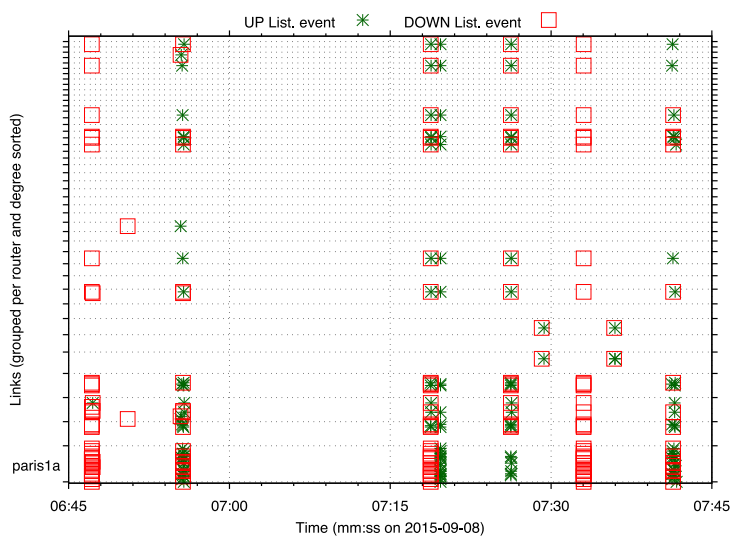

(b) Router-wide flapping (Paris)

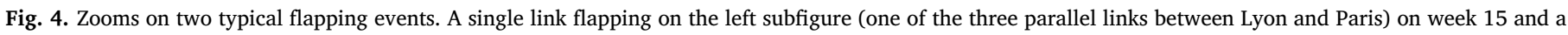

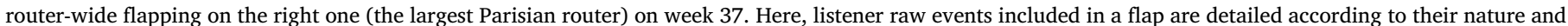

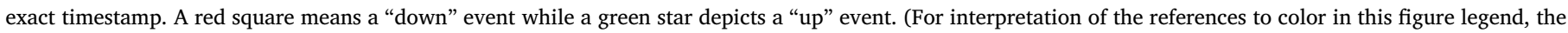
reader is referred to the web version of this article.)

than events belonging to flaps. Regular outages last longer, the median is almost $500 \mathrm{~s}$ and their inter-occurrence last several days, the median is more than 10 days. One feature of these curves, especially the orange ones, is the presence of dense and almost vertical parts. They probably correspond to various protocol timers that flaps stress and so exhibit: lower level link-state triggers, various IS-IS timers such as Hello hold 


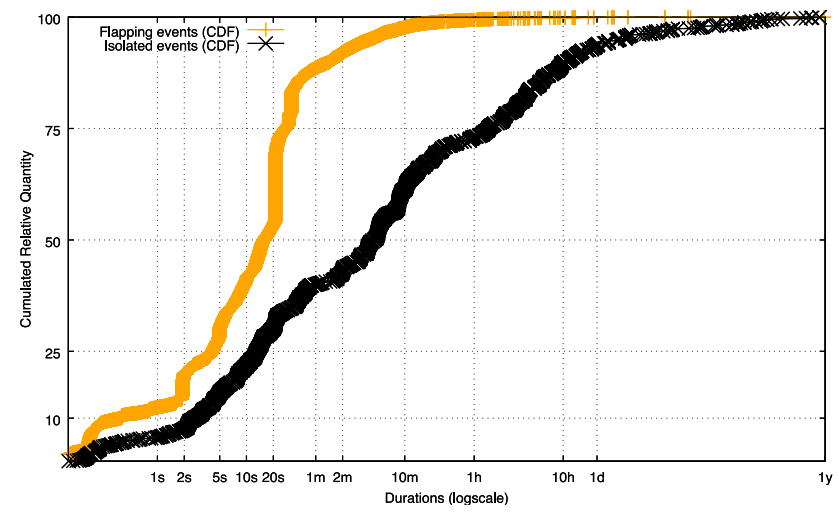

(a) (intra-)outage duration

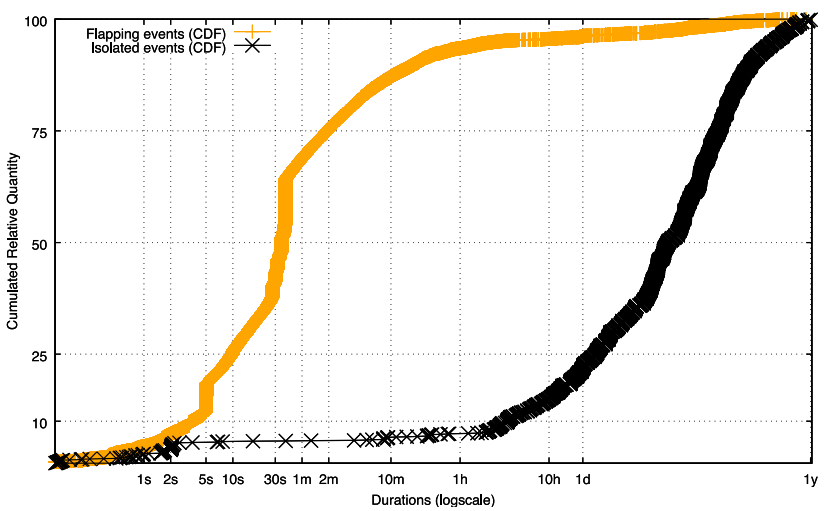

(b) inter-outage duration

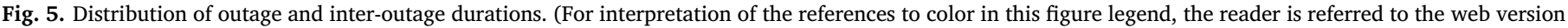
of this article.)

time, LSP generation interval, LSP transmission interval, and so on.

To summarize, our listener has shown that the topology of RENATER is very dynamic. On average, less than 2 days per week are totally free of routing changes. These changes range from a single isolated outage per day up to several thousands recurrent raw events which can be aggregated in intense flapping sequences representing $95 \%$ of all routing events. The characteristics and the root causes of these two kinds of events are distinct, i.e, they range from short fail stop failures to long periods of state oscillations probably due to both software and hardware problems. Performing correlations with other sources of data will help us to better understand these phenomena and their effects on the user traffic.

\subsection{ISP Tickets: manual reporting is not enough}

Collecting this second passive data source is more lightweight than the former but the information we get is less accurate. Tickets are filled in manually, and time unit is often the minute. There exists a risk of errors, approximations and omissions. However, tickets have the strong advantage to provide information on the management plane, notably about planned operations such as maintenance. Ticket parsing is performed by a carefully crafted program, coping with irregularly shaped messages. For a given ticket, only the closing message is taken into account: it provides the temporal period of the operation.

Table 4 provides several insights about ticket characteristics. We observe that incident and maintenance tickets occur roughly at the same frequency, but maintenance events have a longer duration, possibly because maintenance windows are overestimated and their bounds are often rounded to the nearest hour. Tickets cannot be directly compared to listener events. First, for isolated events, a single outage such as a link down then up generates 4 raw listener events for a single corresponding ticket. Second, a long flapping sequence may generate hundreds of listener events which can be summarized by the NOC in only one ticket. That said, it seems that many high level routing events

Table 4

The ticketing system permits to distinguish planned from unplanned operations.

\begin{tabular}{llll}
\hline Tickets statistics (per day) & & & \\
\hline Frequency per type & Average & Max & Total \\
Incident & 0.78 & 5 & 384 \\
Maintenance & 1.03 & 9 & 509 \\
\hline \multirow{2}{*}{ Duration per type } & Average & Median & Max \\
\hline Incident & \multirow{2}{*}{$16 \mathrm{~h}$} & $4 \mathrm{~h} 15$ & $31 \mathrm{~d}$ \\
Maintenance & $4 \mathrm{~d}$ & $6 \mathrm{~h} 01$ & $377 \mathrm{~d}$ \\
\hline
\end{tabular}

are not reported into any ticket. For example regarding only isolated events (see Table 3 ): even the average of $8.47 / 4 \approx 2.1$ isolated outages per day is by itself greater than the average of $1.03+0.78 \approx 1.8$ tickets we collect per day. It is approximatively the same for flap events considering link flapping as bidirectional events: $4.32 / 2 \approx 2.2$.

While maximum values for ticket duration illustrate the issues we can encounter to handle ticket informations, median values seem more realistic as they often cover flaps as outages. Indeed, from the one hand, one maintenance ticket reports an operation of 377 days, while, on the other hand, the median magnitude of several hours is compliant to the average value of flapping outages given in the previous subsection.

To summarize, if tickets can provide valuable qualitative information such as the nature of an event (e.g. planned maintenance window or unexpected incident), ticket count reveals that they are far from covering all routing events detected by our listener, even when considering high level outages. We thus leave any systematic correlation analysis with tickets out of the scope of this paper although we do not notice significant inconsistency between tickets and flap events when they intersect. Look at the appendix for more details. Many routing events (and their corresponding downtimes) are simply unreported. Most of them do not imply a sufficiently long connectivity loss, thus are unnoticeable with basic tools and not subject to a notification to network users. Tickets often report problems which are detected sooner by the DCART listener, this suggests that DCART could help to diagnose problems before they provoke a network outage resulting in a noticeable downtime. Our preliminary analysis shows that an ISP may benefit from DCART with automatically pre-fill incident tickets to improve their accuracy.

\subsection{Active probing: about packet loss}

Active probing is precious since it gives information on the data plane, i.e. the quality of service experienced by customers. The lifespan of our probers was limited to 16 weeks of data as shown on Table 1 . Nevertheless, on this short period, we collected really useful knowledge about real downtime periods in term of data plane convergence. Indeed, the listener is not able to show the impact of convergence delay on user traffic as it does not take into account data plane related issues but only control plane informations. We will use here the term of downtime instead of outage to depict the loss sequence period and duration.

At a global scale, the overall loss rate is very low, i.e. a ratio of $7.6 \times 10^{-5}$. We consider here the cumulated loss periods divided by the period where our probers where effectively able to analyze their own $15 \times 5$ directed flows at maximum. This ratio includes both downtime periods provoked by forwarding changes and congestions that can be due to DDoS or simply traffic peaks. RENATER is robust enough to support most of the failures that occurred. Table 5 provides more 
Table 5

Active data, towards data plane information about packet losses and microloops.

\begin{tabular}{lcll}
\hline \multicolumn{1}{c}{ Average } & Median & Max \\
\hline Packet Loss statistics (per day, per flow) & & \\
Loss rate (\%) & $7.6 \times 10^{-3}$ & 0.0 & 17.14 \\
Duration (s) & 1 & 0.2 & 14,274 \\
\hline
\end{tabular}

“TTL Exceeded" message statistics (per day, per flow)

\begin{tabular}{llll}
\hline Loop rate (\%) & $1.4 \times 10^{-5}$ & 0.0 & $7.8 \times 10^{-3}$ \\
Duration (s) & 0.42 & 0.2 & 10.2 \\
\hline
\end{tabular}

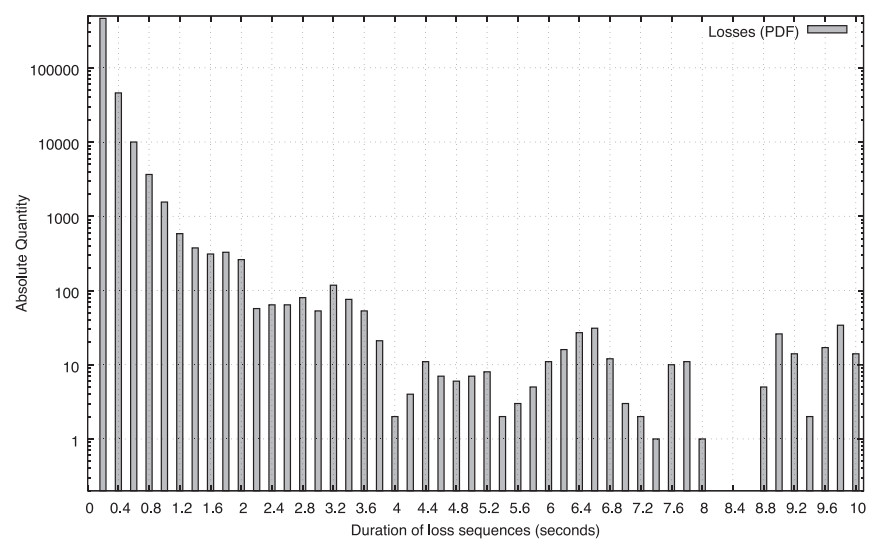

Fig. 6. Distribution of loss durations.

statistical information including typical downtime durations. In particular, we observe that the vast majority of downtime periods consists in a single packet loss while very few events last more than $10 \mathrm{~s}$. The median is of $200 \mathrm{~ms}$ as probes packets are spaced by this constant time and we observe a downtime longer than $5 \mathrm{~h}$ at worst, i.e. the network was partitioned due to a single point of failure, typically a stub router.

In Fig. 6, we plot the detail of the duration distribution of loss sequences observed with active probing, i.e. loss of consecutive packets in the same flow. Some of them are quite long but generally the network seems to converge in less than a few seconds. The vast majority (we use a logarithmic y-scale) consists in just one packet loss and do not necessarily result from a state change but rather from a congestion (e.g. due to a traffic peak, a faulty link or a DDoS attack). The underlying network physical graph looks redundant enough as it is very rarely partitioned: while link outage duration often lasts some minutes, the downtime is almost always less than $10 \mathrm{~s}$. While the average duration is about one second, with no surprise the median of the flow downtimes is only $200 \mathrm{~ms}$ since it is the period we calibrate for sampling. When removing outliers, i.e. single packet loss or long sequences greater than 15 min due to a network partition that affects a given prober, the downtime average is about $0.8 \mathrm{~s}$. In the next section about data correlation, we will see that almost all downtimes greater than 2 or 3 packets coincide with routing changes and that the convergence time is a bit longer than estimated here.

On average and for each prober pair, more than 5 days and half per week are free of any loss: only $18.34 \%$ of days come with at least one loss sequence (busy day). At a global scale, however, losses occur every day and still more than 4 days per week considering only loss sequences longer than a second. This ratio is comparable to the number of busy days with routing changes. Durations of collected losses follow a powerlaw distribution, the vast majority of them are very short, probably due to congestion, only $0.6 \%$ last strictly longer than one second. More surprising, given the robustness of the underlying network, is the significant impact of longer losses. Table 6 shows that although long loss sequences seem to be marginal, they account for $16 \%$ of all lost packets
Table 6

Loss sequence duration and packet loss.

\begin{tabular}{lll}
\hline Loss counter $\backslash$ Loss sequences & $\leq 1 \mathrm{~s}$ & $>1 \mathrm{~s}$ \\
\hline Sequences & $99.4 \%$ & $0.6 \%$ \\
\#Packets in sequences & $84 \%$ & $16 \%$ \\
\hline
\end{tabular}

and have a significant impact.

\subsection{Routing error messages: about loops}

Finally, we also collect forwarding error messages sent back by routers that are triggered by our specifically crafted probes. In particular, DCART records more than 4000 ICMP "TTL Exceeded" messages indicating a significant amount of forwarding loops. Some of them last more than several seconds. However, we do not observe any ICMP "Destination Unreachable" messages. Rate limiters are not configured in the same way for these two kinds of error messages. While replies of the former kind are typically filtered in the shape of burst (token bucket) the second kind of filter is more severe, e.g. 1 message per second per prefix for all traffic [6]. As already mentioned, during convergence of the routing protocol, at a given time routing loops may only occur between two neighboring routers if IGP weights are symmetric [7], each one sending the traffic to the other for a given prefix. The TTL included in the IP header of the probe is decreased by 2 at each round in the loop. When the TTL reaches 0 , the router sends an "TTL Exceeded" to the sender. If the loop occurs inside an MPLS tunnel (which is an usual case in RENATER), the error message is first sent to the tunnel end, the Egress edge router [8] before it is returned back to the prober. Therefore, the error message may itself be caught in the forwarding loop or in a black hole and may never reach the error logger of the sender. Due to both this phenomenon and rate limiting, loop detection is only partial, while all packet losses are detected and all control plane events are received. Note that loops of short duration may also remain undetected because either no packet probe enters the loop or the loop ends before the probe TTL expires. Since probe packets are sent with a small initial TTL (e.g. alternatively 20 and 21), only very short loops are missed. In this case, no packet is lost during the loop. In this paper, we mainly focus on loops triggering packet loss (such that they are already counted in the previous subsection), hence on sufficiently long loops.

We aggregate "TTL Exceeded" messages to mitigate such limitations and so produce loop sequences. First, we do not take into account the reporter of the error messages (the router where the packet expires) but only the flow identifier, i.e. the couple of probers - sender and receiver - and the flow number. Second, we attach loop indications to the loss sequences they belong to. DCART can easily perform this attachment between losses and loops because it parses error messages to extract the quotation of the faulty packet containing the flow identifier. Thus, we do not rely on any time based correlation to perform this deterministic attachment. The duration of a loop is computed as the largest time distance between error messages belonging to the same loss sequence. In practice, it is worth to notice that we never observe long loop sequences where collected error messages are separated by 2 second or more. As we will show later in Section 6, long lasting loops may be complex phenomena involving more than two routers in a cascade of sub-loops.

Table 5 provides some basic statistics about loops. The first observation is that loops seem to be rare side effects of routing changes although some of them last longer than expected. The average is about $400 \mathrm{~ms}$ and we observe several loops lasting more than one second (with a few lasting more than $10 \mathrm{~s}$ ). Analyzing the data at the day granularity offers another perspective on loop prevalence. For a given probing pair, still less than $4 \%$ of days are subject to loops. However considering all flows together, almost one day over two is subject to 


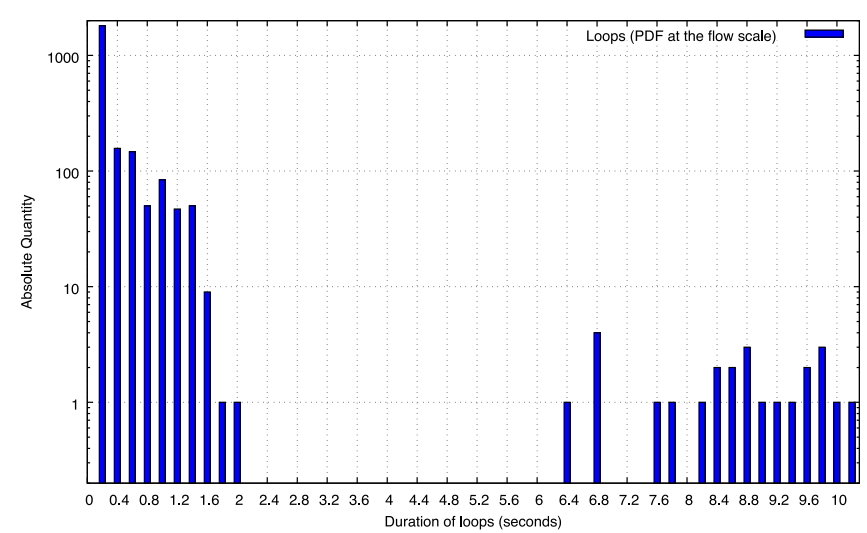

Fig. 7. Distribution of loop durations. (For interpretation of the references to color in this figure legend, the reader is referred to the web version of this article.)

loops, and one day per week is subject to loops lasting more than a second. Since some loops are not detected, these statistics show that their impact should not be neglected. While all loops are attached to a given loss sequence, the vast majority of loss sequences are not associated to loops except when they last long enough, $25 \%$ of those lasting more than a second are attached to loops.

The distribution of loop duration is shown on Fig. 7. Although the vast majority of loops last clearly less than $2 \mathrm{~s}$, some of them last up to $10 \mathrm{~s}$ in very rare occasions. As already stated, their number is far lower than loss sequences, in particular for short ones. Moreover, actual black hole periods reporting the absence of any route for a given prefix are not captured (no "Destination Unreachable" message) but may exist when a failure occurs. In the next section, we will study in depth some possible relations between control, data and management planes to understand, for instance, why loops explain all long downtime periods triggered by up events of the control plane.

To summarize, reported loops are not so rare and negligible events. On average, more than one day over two is subject to transient forwarding loops at the network scale. Even if they account for only a small subset of losses, they represent a significant share of ones that last more than one second. Collected loops last 0.4 second on average and up to $10 \mathrm{~s}$ at worst. These numbers are remarkable considering that the recorded number and duration of loops provided in this analysis are only lower bounds.

\section{Multi-Source correlations: towards the origin of losses}

If individual data sources already provide valuable information on their own, we expect to improve our understanding of dependencies among them from an analysis combining information at multiple planes. In this section, we correlate three sources of data provided by DCART. Our aim is to explain the root causes of losses. Such correlations are performed on a time basis. In practice, we simply consider a maximal time distance. Then we simply attach each loss sequence to its closest routing event in the defined time range. In the following, we will explain how we compute such a time distance to mitigate false positives and negatives. Convergence related losses are generally very close in time to the routing event and their durations are longer than the ones of other (random) losses. While routing transitions may cause packet loss, it is also possible that a high loss rate on a link may induce a routing transition: the link goes down. In this case, the high loss rate will disappear shortly after the link goes down, and appear back when the link goes up if the problem has not been solved: the link may flap. Finally a high loss rate due to congestion (e.g. DDoS) may occur without any routing transition. In the following we will try to distinguish these situations.

In theory, the temporal correlation could be reinforced by additional spatial information provided by the listener. Indeed, DCART has the ability to check whether it is likely that a given loss can be attached to a given link state change considering the possible paths for a given prober pair (this set of paths forms an acyclic graph). However, exactly assigning a given data flow to a given physical path requires to know the hash function in use in ECMP routers. Besides, performing extra topological tests may also lead to produce false negatives. A change on a given router may impact flows that are not directly concerned by the link where the change occurs - we will provide such an example in the next section. These two limitations can either produce false negatives or turn the test in a shape that is not restrictive enough (if the acyclic graph is a large partial graph of the initial network). To summarize, we prefer to encounter a limited subset of false positives (attach data that may not be correlated) than the opposite (miss data which are really correlated, i.e. false negatives) and so only perform temporal correlations.

\subsection{Losses and routing: some background}

Losses may have many causes. Some may occur independently of any problem on the data plane or control plane. This is the case of losses induced by congestion, and among them losses due to DDoS attacks: packets are dropped when router buffers are full. Some other losses may be due to a failing network component. This is the case when packets are dropped because of transmission errors, or because a router has no working route to the packet destination. In the latter case, if the network redundancy can provide alternative routes, this should last only until the alternative route is computed and installed. Finally, any transition in the control plane, be it the consequence of an unplanned state change of a given device or of a new configuration operated by the NOC, may also induce forwarding loops or routing blackhole both provoking losses. Our aim is to understand the origin of such data plane interruptions: what is the share of losses that result from routing transitions?

To illustrate this, consider first a link that goes down. The neighboring router must first detect the failure: this can be achieved either by the routing protocol itself (e.g. with Hello messages or the use of an extra protocol such as BFD) or lower networking layers (e.g. physical alarms). The logical detection delay depends on various timers, such as the IS-IS holding timer. Then the routing protocol must inform all routers of this event by generating a LSP, which may be delayed by various timers (e.g. LSP gen interval) in order to limit the number of LSP generated. These LSP are then flooded to all routers. This is the information we collect with our listener. Then, each router schedules the computation of a new routing table using a Shortest Path First (SPF) algorithm. This computation may again be delayed by the SPF interval timer. Finally, the newly computed routes must be inserted into the $\mathrm{FIB}^{7}$ and $\mathrm{LFIB}^{8}$. A blackhole period starts when the link fails and ends when the FIB is updated, while the associated listener event we collect is somewhere in between. A forwarding loop may occur while FIBs of routers along the path are not consistent. Since the whole updating process is time consuming, it is expected that loops will start a while after the LSP flooding, hence the listener event. So, at the link down, we expect that a loss sequence will start before the timestamp of the listener event and finish after this event, possibly ending with a loop.

By contrast, when a link goes up, alternative paths are used until the update of the FIB, so there is no blackhole. However, losses can be induced by forwarding loops while FIBs are being updated. Therefore, we expect loss sequences to appear after the corresponding listener event, and mostly in the case of loops. In order to associate these loss sequences to the corresponding listener event, we introduce a loop indicator condition: a loss sequence associated to a loop occurring after a

\footnotetext{
7 Forwarding Information Base.

${ }^{8}$ Label Forwarding Information Base, used in MPLS networks.
} 


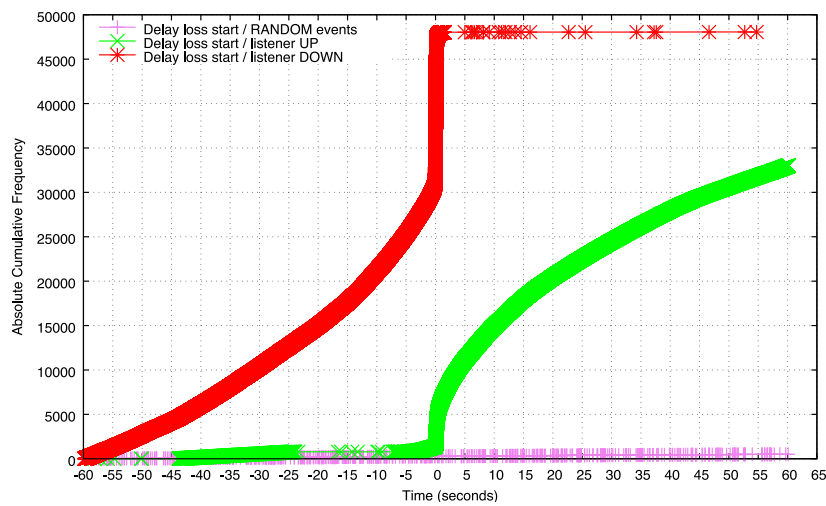

(a) Losses around all listener events (CDF)

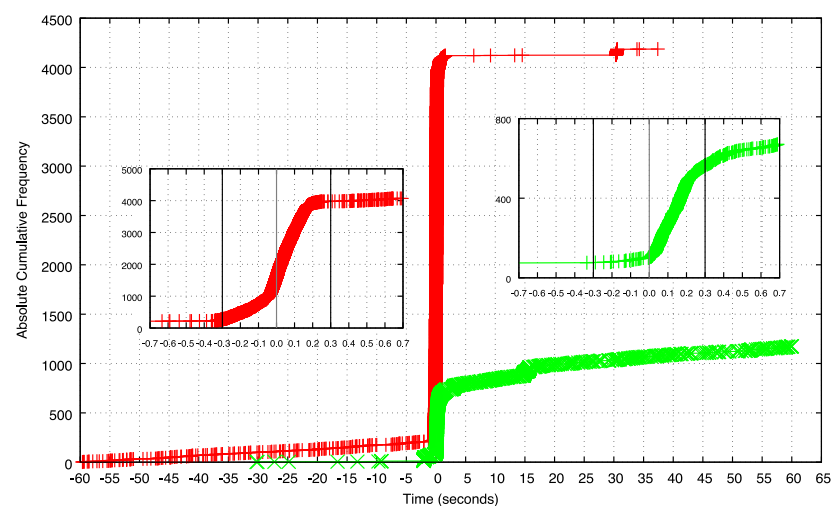

(b) Losses around isolated listener events only (CDF)

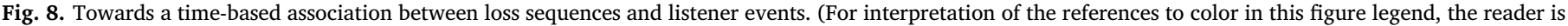
referred to the web version of this article.)

listener event is still associated to this listener event even if our time distance threshold is not verified (in practice, we do not collect any loop indicator after a delay of $2 \mathrm{~s}$ ). Finally, note that the blackhole occurring at a link down will create losses on all flows going through it, while a loop generally only impacts a subset of these flows (i.e. they appear only for a subset of destinations that are not updated in a consistent order among routers).

\subsection{Which time range for the correlation?}

In order to determine the time distance we should use to correlate routing events and losses, we plot in Fig. 8 the cumulative density function of the delay between all listener events and the closest (before or after) losses using a relatively large time window of [ $-1 \mathrm{~min},+1 \mathrm{~min}$ ]. Red (resp. green) point-lines are delays between all attached losses and their closest listener down (resp. up) event. Violet point-lines are randomly chosen reference points within the measurement period in order to show the "normal" loss state of the network when no routing change occur. A loss is actually a sequence, i.e. we define it as a time interval counting the number of loss packets in a row, while a listener event is just a single date. By closest distance, we mean the one minimizing the time between the listener event and the beginning of the loss interval. It is important to understand that while we are particularly interested in losses that follow the actual link state changes, we may face many time shifting between the actual physical change, its recording by the listener and the data plane impact trace (typically a packet loss) it may result in. Several limitations make the challenge more complex than expected: NTP synchronization accuracy, the delay between the real physical change and the signal it triggers towards the control plane, LSP processing durations, transmitting timers and, finally, the sampling frequency tradeoff of our probers. Therefore, and in practice, DCART may record a loss sequence before its associated listener event even if it is its consequence.

Each sub-figure aims to show a different perspective: while all losses are considered on Fig. 8(a), we remove those attached to flapping events on Fig. 8(b). Accordingly, note the sharp decrease in the number of loss sequences on the y-axis, due to the prevalence of flapping. The comparison between these two sub-figures is really useful to understand the impact of flapping on our dataset: when looking only at Fig. 8(a) one may notice that an impressive quantity of losses are relatively close in time to routing events but no clear threshold arises. The shape of the green and red curves is still significant for values respectively close to $+60 \mathrm{~s}$ and $-60 \mathrm{~s}$. It means that such losses do not really result from the convergence of the routing control planes, but are due to lossy links because of either bad transmission quality that may trigger link flapping, high congestion rates as DDoS or random traffic congestion. The difference with the loss pattern around random points (violet curve) reinforces this strong correlation between flapping and loss sequences. The symmetry between up and down events also shows that those losses occur at the same level of frequency after up events and before down events. This is to be expected: on a lossy link, losses appear when the link goes up, and disappear when the link goes down again, see Fig. 14 for a detailed spatiotemporal view.

It can be seen in Fig. 5(b) that, even on a flapping link, the delay between an up and the next down (inter-outage) is greater than $5 \mathrm{~s}$ in more than $90 \%$ of the cases. It is thus unlikely (although always possible) that a loss caused by an up event be closer to the next down event on the same component. When looking at Fig. 8(b) a very tight transition arises around 0 second. The two small zooms giving more precise thresholds, we can see that a vast majority of loss sequences start in the range $[-0.3 \mathrm{~s},+0.3 \mathrm{~s}]$. Experimental bounds to use clearly appear as no flapping noise disturbs data. We use them to extract losses that we consider following the routing change even if the routing event is included in a flap. This tight range helps us to remove most bursty losses around flapping periods from the one following routing changes and typically lasting longer. Most of other packet losses are short sequences, i.e. a single packet, but result in a significant loss rate on long periods of time. Clearly, down isolated events are correlated with more losses than up events.

The red curve (losses at down events) starts to grow at $-0.3 \mathrm{~s}$ and has mostly reached its maximum at $+0.3 \mathrm{~s}$, while the green one (losses at up events) starts to grow a little later, and continues to grow slowly after the $+0.3 \mathrm{~s}$ point as expected. A closer look into the DCART database shows that a significant share of those late losses sequences are actually associated with "TTL Exceeded" error messages. This indicates that these losses are associated with a forwarding loop associated in its turn with the last up event. This is why, in the following, we also consider the loop indicator condition to correlate listener events and losses, even if they occur one second after the $+0.3 \mathrm{~s}$ threshold.

\subsection{Listener events vs. loss sequences}

Before performing fine grained time based correlations based on the previously defined time distance, we first give a coarse chronological picture of losses and routing events to enable a basic comparison between our various data sources. Fig. 9 shows two raw representations of losses and listener events both in space (by couples of probers) and time: routing events are drawn in red (down events) and green (up events) dotted lines, regardless of their location, while losses are represented as black squares on the line corresponding to the couple of probers where they occurred. Couples of probers are grouped according to the sending prober. For example, the first set of lines above Besançon (see y-axis) represents the couples where the sender is Besançon and the receivers are Caen, Cergy and so on, with the same order as senders. 


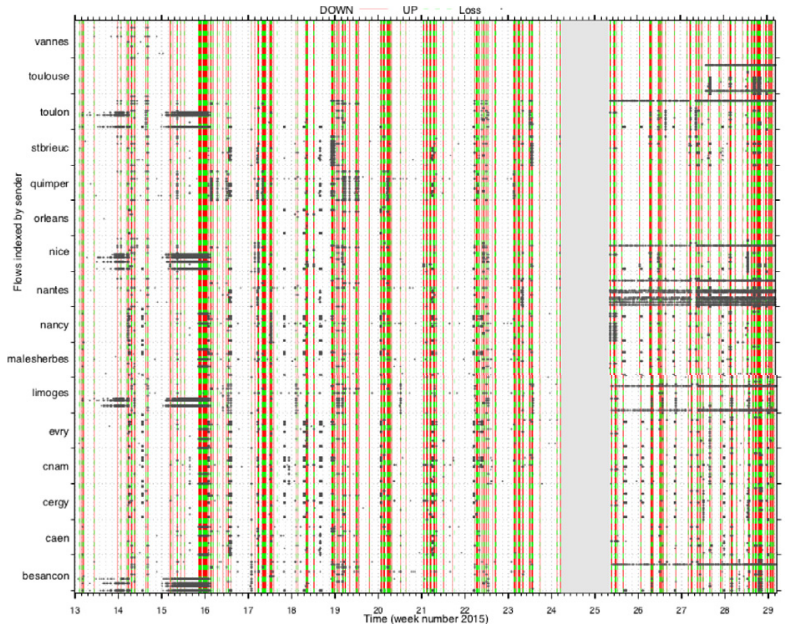

(a) All losses (one packet or more)

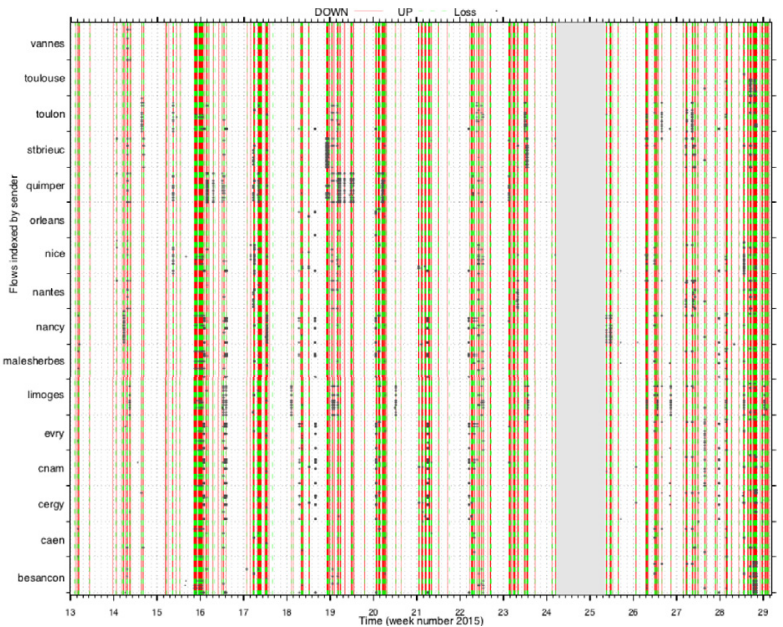

(b) Losses with at least three packets in a row

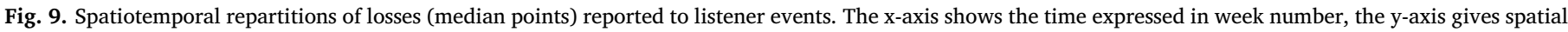

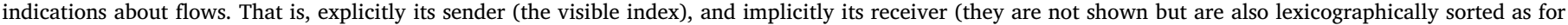

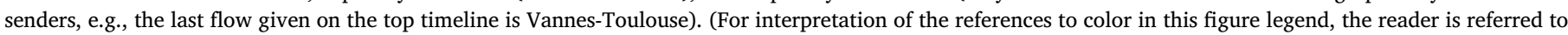
the web version of this article.)

Fig. 9(a) shows all losses, whereas Fig. 9(b) shows losses lasting more than $400 \mathrm{~ms}$, that is at least 3 lost packets in a row. Note that the large vertical gray rectangle starting at the end of week 24 indicates a two weeks period where data are not available due to probers down time.

Comparing these two figures, we can see that longer losses are much more directly associated with routing events than short losses as most of loss events meet listener events in Fig. 9(b), on the contrary to Fig. 9(a). For example, the rather long black lines for Limoges during week 15 in the former view, crossing periods without routing events, disappears in the latter view. Indeed, this kind of loss pattern is a first hint that a link is faulty: the error rate is under the radar and is not correlated with routing events contrary to higher error rates. Similar black lines simultaneously appear with several senders, Besançon, Limoges, Nice and Toulon, showing that these flows cross the same faulty link (ParisLyon). Obviously, short losses may also be due to congestion independently of any routing event. A lossy link seems then to be one of the main root causes of listener events (and flaps in particular), as we will see on Fig. 14 and discuss in the next section. A low error rate yields a random packet loss pattern with short loss sequences. The control plane reacts above a certain loss threshold, either at the routing layer (Hello or BFD Messages) or at lower layers (e.g. SDH alarms or L2 triggers). These visually long loss periods do not consist in long loss sequences but rather in a great number of one or two packet loss sequences: the threshold of three packet losses in a row of Fig. 9(b) is not reached. On this figure, for example at the fifth day on week 18 or the second day of week 22, we observe that some non correlated long losses occur several times with the same set of destinations. Such losses are severe congestions due to DDoS attacks ${ }^{9}$ : in practice (look also at Fig. 9(a) to find the same patterns with all kind of losses), this part of the network has been regularly attacked during the period of monitoring and provokes many rather short losses, from many one packet losses up to quite rare four packet losses.

Using our simple attachment model for correlating routing changes and losses that follow, we can dig into real networking questions. First, what about the share of listener events correlated with at least one loss sequence? On the active collection period, we determined that about $64 \%$ of the more than 1900 down events are closely related to losses and, more surprisingly, about $55 \%$ of the same number of up events also result in losses. Without the loop indicator condition, this

\footnotetext{
${ }^{9}$ As it has been confirmed by an administrator or RENATER.
}

percentage falls to $40 \%$. Note that these numbers are lower bounds on the number of listener events correlated with losses. DCART records all listener events but does not collect losses on all possible paths: probers cannot cover all existing paths in the network in particular because of the path diversity due to ECMP and parallel links. Moreover, since we do not detect all routing loops, the loop indicator also gives a lower bound. So, as a first conclusion, we can say that a vast majority of listener events come before losses, and this both when links are going up or down.

Let us now focus on the severity of losses related to listener events to better understand the kind of root causes that may explain such surprising results. We plot on Fig. 10 the longest correlated loss sequence for each down and up listener event (using the loop indicator). We then plot the distribution of those worst cases for each listener event using a logarithmic y-axis.

Most loss periods are rather short, lower than $2 \mathrm{~s}$ in average when removing outliers, especially for up events. However, we observe that down or up events provoking loss periods of several seconds are not negligible and can impact critical traffic. Moreover, we notice that there exists a small amount of up events producing very long loss periods. We will see later with Fig. 13 that they result from long forwarding loops. Indeed, it is unlikely that a simple traffic shifting may produce such long term and severe congestions.

Routing-caused losses are longer on average. While we compute an average of $14.4 \mathrm{~s}$ (to be compared to the mean of only $1 \mathrm{~s}$ observed in Section 4.3) when considering outliers ${ }^{10}$, the mean is about 1.5 second without (to be compared to $0.8 \mathrm{~s}$ in Section 4.3). The comparison between the event type (up/down) is rather clear on the figure. Down events generate much more longer losses than up events. They are worst both in terms of average duration and quantity. We compute the following respective means for each kind of events (including average and medians with outliers in parenthesis): $1.2 \mathrm{~s}(0.7 \mathrm{~s}, 0.4 \mathrm{~s})$ for up events and $1.8 \mathrm{~s}(18 \mathrm{~s}, 0.4 \mathrm{~s})$ for down ones. The existence of a blackhole period at down events explains these not really surprising differences.

After looking at the consequence of routing changes, we will take a look at the opposite question: what is the share of loss sequences correlated with listener events? On Fig. 11, we focus on the complete set of

\footnotetext{
${ }^{10}$ That is either single packet losses that are difficult to interpret since it is directly related to our sampling rate or losses longer than $15 \mathrm{~min}$ that are due to the disconnection of a given prober.
} 


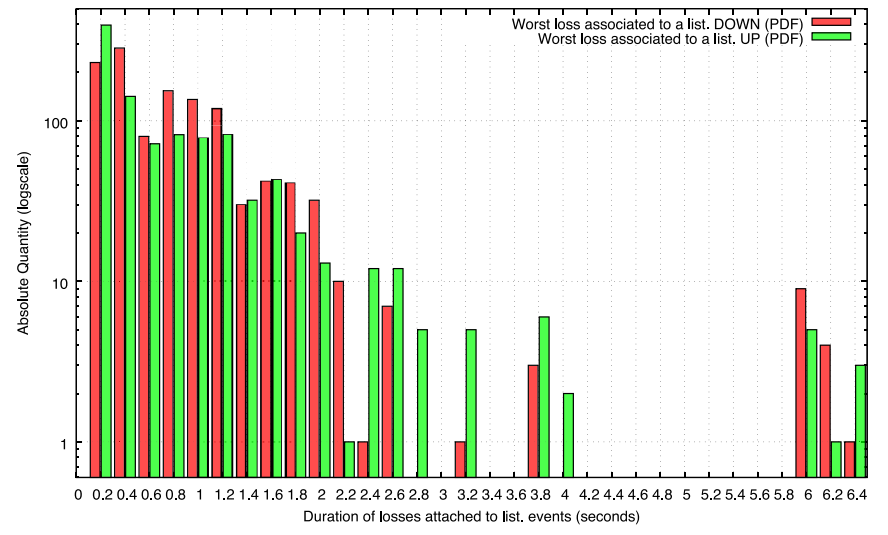

Fig. 10. Distribution of the duration of the worst loss sequence correlated with each listener event. (For interpretation of the references to color in this figure legend, the reader is referred to the web version of this article.)

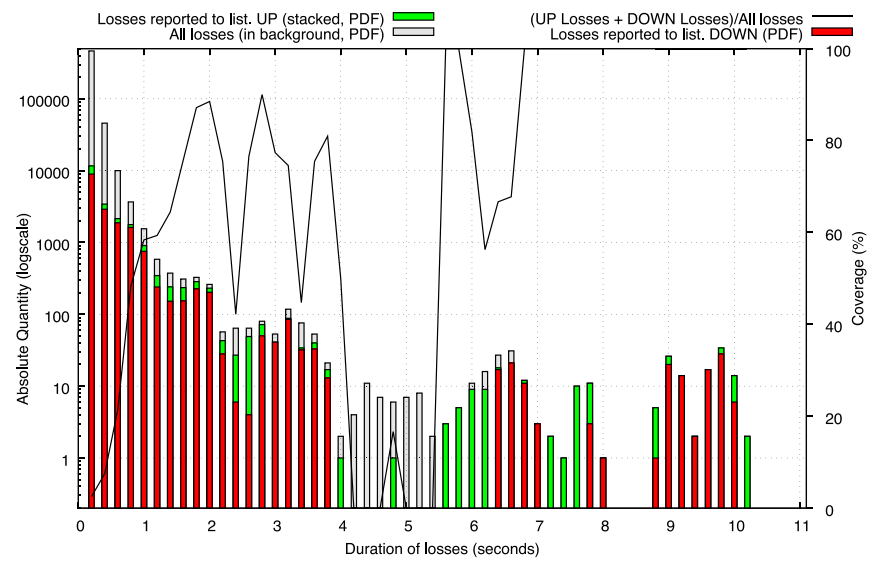

Fig. 11. Distribution of loss durations and share of losses directly following listener events (sorted according to their durations). (For interpretation of the references to color in this figure legend, the reader is referred to the web version of this article.)

losses shorter than $11 \mathrm{~s}$ (since longer loss sequences are really rare) and the subset of losses correlated with a listener event. Compared to previous results, it means that we do not consider here the number of listener events but the number of losses which is two orders of magnitude higher. Thus, the same listener event may be associated to loss sequences on many prober flows. We plot the distribution of the duration of all losses (grey boxes), those correlated with an up (green boxes) or to a down event (red boxes). Red and greens boxes are cumulative while the grey one is plotted in the background. Since the logarithmic scale used on the y-axis makes comparisons difficult, we also plot the ratio of losses related to a listener event using a linear scale at right. We first observe that very short loss sequences, including only one or two packets in a row, usually do not result from routing changes, they are rather due to short congestions or faulty links.

In contrast, we observe that a majority of longer losses from 1 to $3 \mathrm{~s}$, seems to be strongly related to routing changes. For even longer losses, correlation is not clear to the same extent because the number of such events is very low anyway. Note that while listener events give a precise fault location, losses are less explicit since they may occur anywhere along one of the paths between a couple of probers. As a direction for further investigations, when losses occur at the same time for distinct prober pairs, but are not correlated to listener events, we aim to improve the localization of the fault by intersecting the paths in use for all concerned flows.

We compute that only about $28 \%$ of long loss sequences are not (closely) correlated with listener events. A long loss sequence being defined as strictly longer than $1 \mathrm{~s}$ in the whole paper. A closer look in our database indicates that a majority of them occur on flows going through Paris, Rouen, then Caen. RENATER confirmed us that these links experienced several strong congestions, with throughput above $9.95 \mathrm{~Gb} / \mathrm{s}$ on $10 \mathrm{~Gb} / \mathrm{s}$ links, due to recurrent DDoS attacks on this part of the network. We can conclude that in our dataset, only $\approx 10 \%$ of long losses do not seem to be closely correlated with a routing event or a DDoS. They may result from very rare long congestions, system anomalies of the platform or just being the result of a missed correlation due to a too tight correlation time margin. Indeed, the vast majority of remaining non associated long losses occur in flappy periods. As a final observation, note that the localization of the DDoS attack was possible thanks to the use of several flows between probers: between the same pair, some flows were subject to losses while others, using another path, were not. With a single flow, we could not have localized the problem, or possibly it could have been undetected altogether.

To summarize, we have shown that most $(60 \%)$ routing events are followed by losses, and this is a conservative estimation since DCART has a limited time and spatial coverage and a rather conservative correlation. It is particularly significant to note that $50 \%$ of up events are followed by a loss, while there is no loss of physical connectivity. Without the loop indicator condition, this percentage falls to $40 \%$ which is still important, and illustrates that at up, loss sequences are delayed after the listener event, while this is not the case at down time. However losses following a down event last longer and affect more flows than those following an up event. $72 \%$ of long losses (i.e. $>1$ s) clearly resulting from routing events, DCART reveals that down events accounts for $60 \%$ of them and up events for $12 \%$ respectively. The remaining ones are mainly due to a recurrent DDoS attacks or flappy links. Correlating short loss sequences (in particular one packet losses) with routing changes is more difficult since they can be the result of many other causes.

\subsection{Loop and loss vs. listener events}

We now start to quantify the amount of forwarding loops when routing events and/or long losses occur. In Fig. 12, we can look at the spatiotemporal distribution of error messages, attached to long loss sequences (each sequence is plotted at its median time). While most short loss sequences are not correlated with loops (those losses are not shown here), looking only at longer sequences, i.e. at least 3 packets lost in a row, a significant share of loss sequences seems to result from loops. However, most of them seem to be loop-free. While some routing

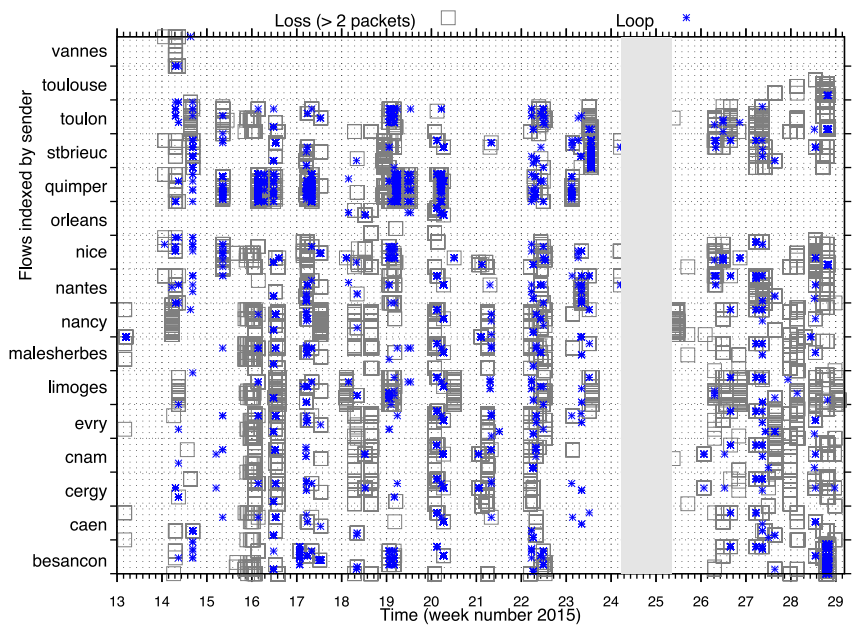

Fig. 12. Spatiotemporal repartition of loop sequences with respect to loss sequences. Loops explain a significant share of long loss sequences and impact many flows at the same time. (For interpretation of the references to color in this figure legend, the reader is referred to the web version of this article.) 


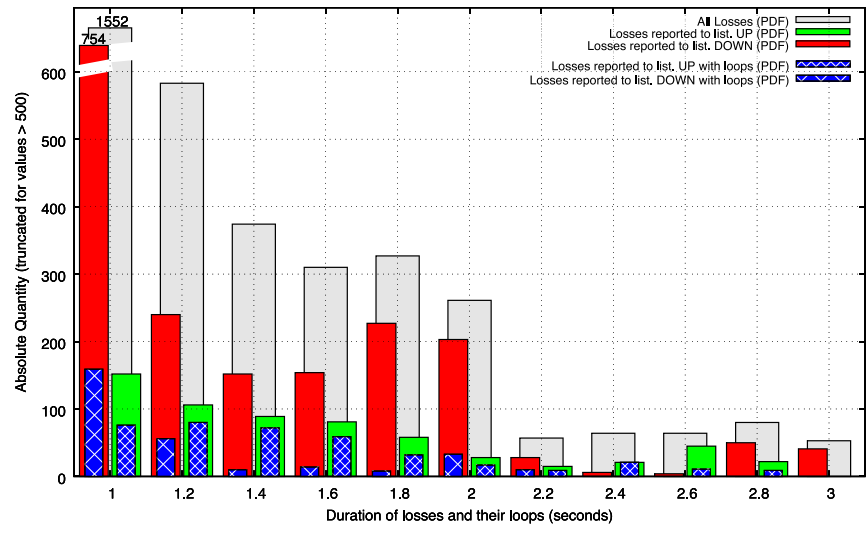

Fig. 13. Distribution of loss durations and share of losses and loops triggered by listener events according to their durations. Loop sequences are attached to their respective loss sequences and this association is correlated with listener events. (For interpretation of the references to color in this figure legend, the reader is referred to the web version of this article.)

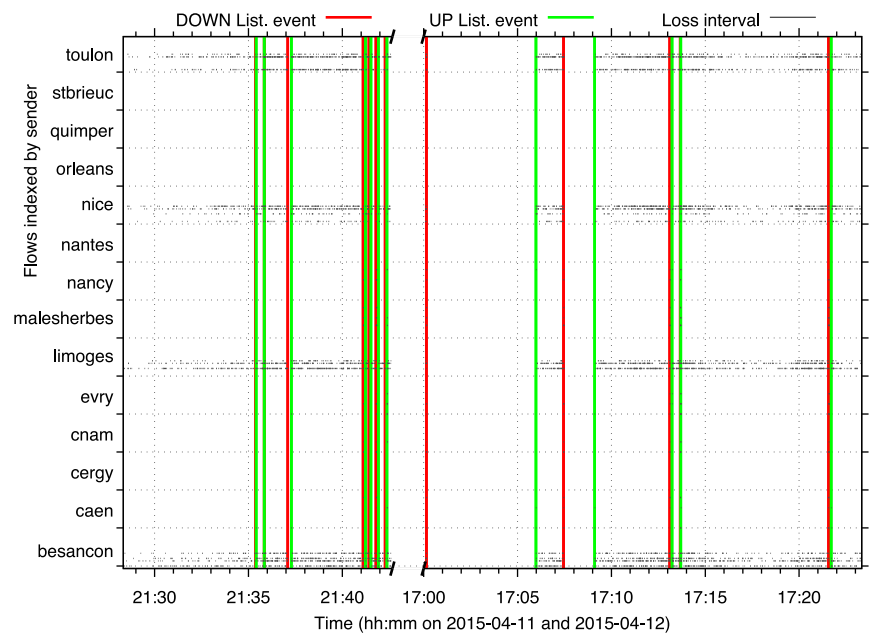

Fig. 14. Spatiotemporal relation between control plane and data plane. Flapping and long lossy periods of one of the 3 parallel links between Paris1 and Lyon1. (For interpretation of the references to color in this figure legend, the reader is referred to the web version of this article.)

changes may appear totally loss free because of DCART sampling limitations to capture very short convergence periods, it is clear that a large share of routing changes do not trigger forwarding loops. Also, one can see on this spatiotemporal plot, that many flows incur both long losses and loops at the same period, grouped in the same column of the graph.

Fig. 13 shows the triple correlation between losses, listener events (either up or down) and, among them, those correlated with loops. Short sequences (less or equal to $1 \mathrm{~s}$ ) are very frequent and are truncated on the figure. One can see that a large fraction of long losses are correlated with listener events, with more downs than ups, as already illustrated in Fig. 11. More interestingly, up events are more often correlated with loops than down events. There are many possible reasons for loops to occur more frequently at up than at down. Globally, and assuming that loops occur mostly on the last link before the link going down or up, if the router $R$ adjacent to the routing change takes more time to update its FIB than its predecessor $P$, it turns in two options. Either, at down, there will be no loop but a blackhole on $R$ that lasts a little longer or, when the link goes up, a loop occurs between $R$ and $P$. There are many reasons for the router $R$ to be slower than $P$ : it
Table 7

Causes of short and long losses.

\begin{tabular}{lll}
\hline & \multicolumn{2}{l}{ Loss sequences durations (\%) } \\
\cline { 2 - 3 } Cause of loss & $\leq 1 \mathrm{~s}$ & $>1 \mathrm{~s}$ \\
\hline Flappy links & 22.9 & 11.5 \\
Blackholes: down / up & $3.0 / 0.5$ & $48.2 / 7.2$ \\
Loops: down / up & $0.1 / 0.2$ & $5.2 / 11.9$ \\
Other (incl. DDoS) & 73.3 & 16.0 \\
\hline
\end{tabular}

has more control plane operations to achieve, such has establishing LDP sessions, and more data plane operations to perform because the closer a router is from the failure point, the more prefixes it has to update. Finally, a link up may be part of a router up that may stress its CPU. We observe that many losses due to an up event appear up to 1 second after the link addition while losses correlated with a down event are much closer in time around the failure and frequently just before rather than long after.

Performing control vs. data plane correlations provides us with many results and the ability to classify losses according to their causes. Although long loss sequences of more than 1s are quite rare, we recall that they account for a significant share of lost packets, around $16 \%$, as seen on Table 6. Most of these long loss sequences can be explained by correlated routing transitions. A summary of the origin of losses is given in Table 7. In this table, loss sequences are classified as Flappy links if they occur during a flapping period, and if they are not associated to a link transition. Loss sequences associated to a transition are classified into loops if a loop has been detected or blackholes otherwise. Note that the tight time margin we use for validating a correlation between a loss and a routing transition may result in an overestimation of losses associated to flappy links in particular for long losses. On the contrary, we observe that many short losses may occur before the actual detection of a flappy link resulting in an underestimation of the share of them actually correlated to flappy links.

Flappy links account for a significant share of losses in general but long losses are mainly the result of routing transitions. Almost half of long losses results from blackholes occurring at link down and a significant share of them (more than 10\%) results from loops occurring at link up. A vast majority of short loss sequences are not correlated with routing events (e.g. random errors, DDoS attacks), whereas most long loss sequences are indeed correlated.

From these results, one can envision several more or less automatic solutions. First, about forwarding loops, there already exists solutions to mitigate or prevent them [7]. They do not require any manual intervention nor significant protocol change: it is up to the ISP to decide to deploy them with a very limited overhead. Second, about blackholes, it is also possible to automatically limit such periods by speeding up the convergence time using existing mechanisms such as PIC, LFA, incremental SPF or equivalents [9]. One can also mitigate such periods by configuring several timers that may slow down the convergence or by using more appropriate hardware to quickly detect failures. Eventually, for flap related issues, we do not believe in a fully automatic solution. Indeed, while it is possible to envision some vendor dampening solutions (i.e. shutting down a link that changes its state too frequently), it is a bit risky to automatically remove a link as it can put the network in a degraded state (e.g. partially disconnected in the worst case). Here we prefer to consider the deployment of a support for triggering human actions. That is triggering alarms, that may automatically pre-fill NOC tickets, intended to network administrators. Indeed, before removing even transiently - a flappy link, it is preferable to let the NOC decide on its own which is the most suitable answer: letting some loss occur or taking the risk to disconnect some part of the network. The decision may vary according to several factors e.g., the loss rate, the flap density and the link position in particular. 


\section{Short illustrative stories}

In this section, we show on two examples the ability of DCART to enable fine grained analysis. We have seen in previous sections that a flapping link may be a quite common and perturbing event. Our first example is a long lossy sequence starting on 2015-04-07 in the morning - look at Fig. 9(a) at the end of week 14. The first listener down event occurs in the evening of 2015-04-11, and Fig. 14 illustrates several instances where losses start when a link goes up, for example around 17:05 and 17:08 and stop when the link goes down, for example around $17: 00$ or 17:07. Although surprising at first, it means that there is a faulty link with a high loss probability due to a physical problem. When up, some flows use this link and losses appear while if the loss rate becomes too high, the link goes down, flows are routed through other links and losses disappear. The link may go down at the IS-IS level either because the routing protocol itself detects a problem (loss of Hello IS-IS messages) or a lower level trigger indicates a bad link quality. Note that the first down periods, and most of them in general, last only a few seconds or even less before the first illustrated long outage at 17:00 that lasts more than $5 \mathrm{~min}$.

Moreover the corresponding NOC incident ticket was only opened on 2015-04-13 at 06:47 and reported a hardware problem on an optical component, 4 days after the first loss sequence and more than one day after the first link down event. This case illustrates the possibility for the NOC to anticipate a link failure, before any routing alert and without any monitoring of the link itself. It is also worth to notice that without the ECMP capability of DCART this kind of lossy link may not be noticed by the ISP: it only impacts a subset of the flows going through the bundle between Paris1 and Lyon1. To monitor its network, an ISP performing a simple ping or traceroute that does not go through this specific load balanced path may not detect the problem.

Finally, note a very intriguing phenomenon. Although flows crossing the faulty link incur long lossy periods while the link is up, such as flows originating from Limoges for example, some other flows incur single loss sequences at up or down transitions, such as flows from the Caen or Evry probers towards the prober of Besancon for example, at 21:42, 17:05, 17:09. These tiny black points representing losses are surprising. They imply that a flow that does not go through the lossy link, but via another parallel link between Paris1 and Lyon in practice, is however impacted by this state change, although not to the same extent. This means that when a router updates its data plane for an ECMP bundle, short loss sequences may be induced on all flows going through this bundle. The software forwarding architecture involved for enabling ECMP features on the IOS XR used by the Cisco CRS router of Paris1 seems to provoke a flawed FIB update for the whole bundle.

We give now an even more detailed analysis of a severe link flapping problem inducing loops, losses, and path length changes. Fig. 16 describes the interconnection of the different probers and routers. When the Vannes - Nantes link is up, flows from the Quimper prober towards three destination probers use the green paths. When this link is down, these flows use the red paths. During transition, while routing tables are gradually updated on intermediate routers, routing loops may occur on links with a green edge and a red edge in opposite directions. Fig. 15(a) shows the hop count of 3 flows from Quimper to Toulon (black), Nantes (pink) and Limoges (orange), reported ICMP error messages (blue crosses) as well as listener events (green and red arrows). One can see that at the first listener down event (at 39:47), the hop count of these flows incurs an increase of two hops, the hop count is back to normal at the next up event. Furthermore, loops are detected both at down and up events. One can also observe around time 40:07 that the hop count of orange and pink flows have a small peak. This is because packets were briefly going round in a loop before being released when the loop disappears.

Fig. 15(b) is an even closer look at the last link up event. This time, we analyze the loop locations by inspecting the senders of ICMP "TTL Exceeded" packets and determine the cooresponding flow by inspecting the original IP datagram it encapsulates. Different shapes (triangles, squares, circles) show the loop location. Looking at the flow towards Toulon, one can see a first loop between Brest and Quimper, followed by a loop between Lorient and Vannes. It means that the Brest router converged first, followed by Quimper, followed by Vannes. Lorient converged before Vannes, but also probably before Quimper since no loop was detected between these two, but it is also possible that error messages from Lorient were lost. Meanwhile, this flow incurred a loss period of $1.8 \mathrm{~s}$. Another interesting lesson is that the change in loop position for these 3 flows is not synchronized. For example, the flow towards Nantes is already directed towards Vannes at 40:22.9 while the flow towards Toulon is still being directed towards Brest at 40:23.2, more than $300 \mathrm{~ms}$ later. This means that the update of a router data plane after new routes have been computed is done sequentially for different destination prefixes, inducing different convergence times for different prefixes.

Long loss sequences, in particular the ones related to loops, are a direct consequence of the convergence of the routing control plane and the FIB update. In such cases, loss sequences may last up to a few seconds, in particular when forwarding loops occur. Other loss types, consisting in many short loss sequences, are rather due to a faulty device. Those losses precede the flapping detected at the control plane. However if the error rate is below a given threshold (look for example at the Signal Failure Bit Error Rate feature of Cisco WAN physical controller), those losses may occur for a relatively long period without triggering any routing event. If the error rate goes above this threshold, the link goes down (due to a physical trigger or to the losses of IS-IS Hello messages), and losses disappear. When the quality of the link returns to normal, the link goes up again, and losses reappear. A quality level fluctuating around the critical threshold may induce flapping events (such as the one in Fig. 14).

DCART helps to explain complex scenarii involving some intricate network behavior such as ECMP bundling and priority among prefix updates. More generally, it also shows some subtle and detailed effects of flapping and loops.

\section{Related work}

As an evidence of the importance of monitoring for verifying and improving network performances, a large number of monitoring tools are currently available to ISP $[10,11]$. Those tools typically unify a set of more basic tools for active measurements, from IP SLA reports to SNMP traps, Syslog collection and distributed monitoring probing. Contrary to DCART, however, those tools typically have pre-determined capabilities, which makes the tools inflexible and unsuitable for custom monitoring needs. Moreover, they rarely allow to correlate data from control plane and management plane. This translates into the lack of support for specific analyses enabled by DCART, such as quantifying and classifying the disruptions on user traffic triggered by the routing convergence.

Few research has targeted flexible multi-sources monitoring. Indeed, most previous works focused on specific measurements. Some contributions restricted their analysis to the monitoring of the routing protocol, e.g., [12]. In [13], Shaikh et al. describe an architecture that analyzes the routing state of a multi-area OSPF network. Others, for example in a study of the Sprint's IP backbone $[14,15]$ use more data sources, but only analyze the impact of link failures occurring during maintenance windows on the IGP convergence period (that turns into traffic disruptions for their set of active probes). In [16], Medemet al. propose an algorithm based on document clustering techniques to automatically retrieve relevant information out of manually filled network trouble tickets. They use this technique to correlate IS-IS intradomain routing changes with trouble tickets in [17]. In [18], Wanget al. use ICMP messages to detect loops and packet losses during routing changes. Routing changes are not monitored through a listener but only by running traceroute, and the main focus is on the BGP control 


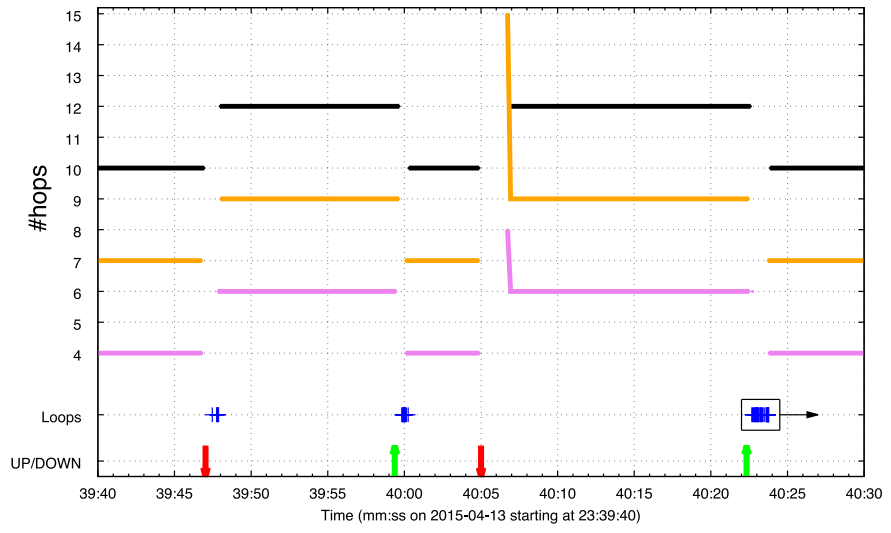

(a) Effects of routing changes on the number of hops

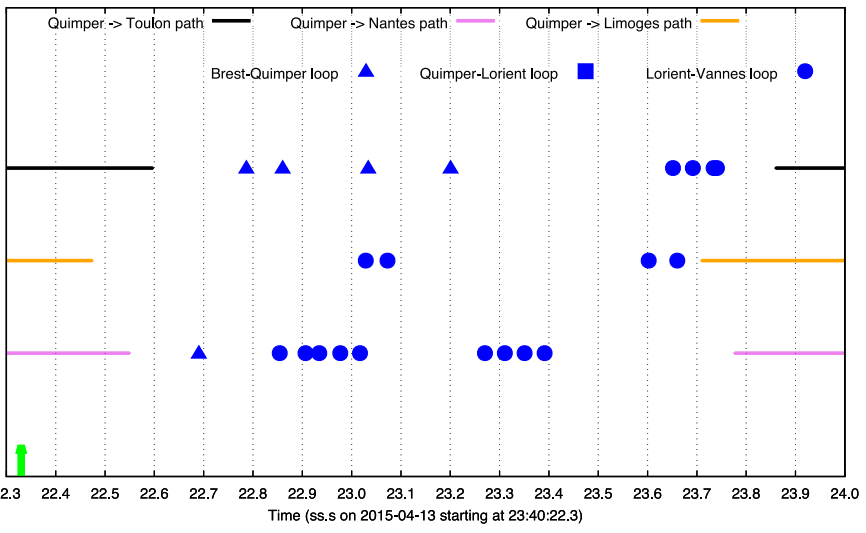

(b) Zoom on the last link up event

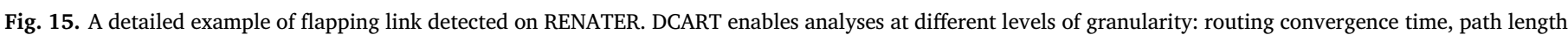

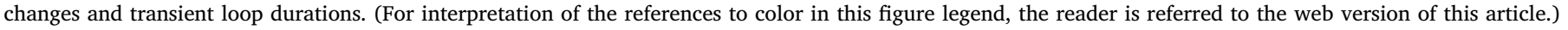

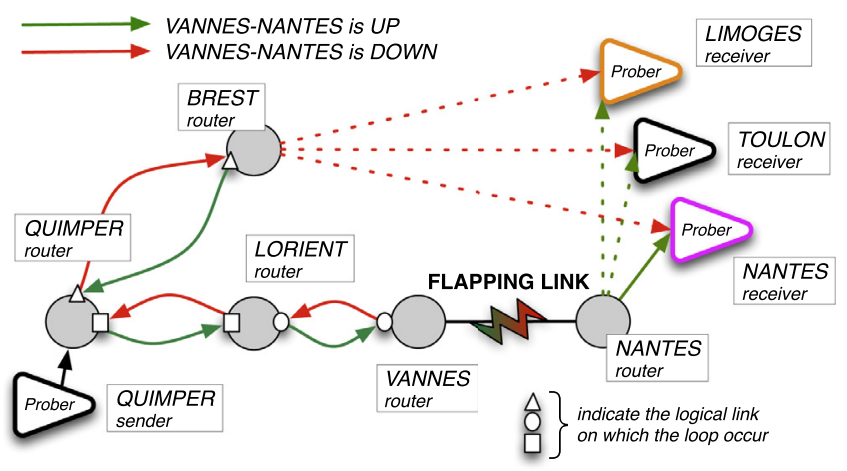

Fig. 16. Flapping link study case. (For interpretation of the references to color in this figure legend, the reader is referred to the web version of this article.)

plane. Similarly Feamster et al. [19] use active probing with traceroute, and BGP messages to evaluate the effect of routing changes. In [20], Myakotnykh et al. use active probing to monitor a single endto-end inter-domain paths, with fine-grained NTP synchronization. Again, routing events are inferred from traceroute measurements. Finally, Barford and Sommers [21] compare active and passive methods for packet-loss measurements but they do not cross them.

NICE (Network-wide Information Correlation and Exploration), presented by Mahimkar et al. [22,23], is the monitoring platform which is the closest to DCART since they use both passive and active monitoring for performing correlations. However, instead of simply using timestamps as the correlation index, they use more sophisticated statistical tool to perform such correlations. Note that authors discuss potential problems with timestamp based correlation, but these problems did not show up in our experiments. In [23], authors study the impact of router maintenance and network upgrades on network performances. In [24], correlations among numerous data sources, including tickets, is done via statistical methods, using multi-resolution analysis. However, routing events are discovered through syslog and there is no active probing involved. Generally speaking, authors of NICE related contributions mostly emphasize their statistical methodology, and the design of their platform in general, rather than providing various and tangible networking results. With DCART we follow another path and provide many networking insights explaining data plane downtimes and hints towards their mitigation as listed in the conclusion.

DCART can be used to integrate data plane, control plane and management plane analyses and correlate their outcomes with userdefined queries. DCART flexibility translates into its ability to systematically perform any fine-grain analyses. As an example, we described in this paper how DCART can provide evidence of transient loops triggered by the IGP routing convergence. Although those loops have been largely discussed and some solutions have been proposed to mitigate their impact [25-27], few works provide actual measurements of their duration and likelihood to occur in real networks. To the best of our knowledge, apart from the work of Hengartner et al. in [28], no real measurement results are available on this topic. For performing this precious analysis, authors propose an algorithm to detect loops from packet traces (i.e. packet captures directly performed on routers), and then actually apply it to the captures collected in a real ISP backbone. This algorithm thus requires passive collection of packet traces, which poses major scalability issues for its application in an online monitoring tool. Collection of huge traces from potentially all routers in the network is indeed required. On the contrary, DCART offers a data flow perspective of forwarding loops which is the first of its kind to the best of our knowledge. It is however interesting to note that our results about loop frequency and duration, although more detailed than the ones they provide, are basically in line with their measurements which have been collected more than 10 years ago on another ISP network.

\section{Conclusion}

In this paper we have shown how DCART efficiently collects and combines multiple sources of information about the network state. Strong temporal correlation can be observed between listener events, packet losses and routing loops. As a first result, we have verified that forwarding loops actually occur during routing transitions. In worst cases, such loops last several seconds. Not only at link down but even, and mostly, at link up operations (observation $i$ ). Another interesting result is that the vast majority of routing events and most loss sequences are related to flappy links (observation ii). In a network with a redundant topology, flapping may go unnoticed. With the ECMP finegrained loss monitoring enabled by DCART, link flapping may not only be detected, but possibly be anticipated with the rise of loss frequency. Finally, blackholes, i.e. periods where there is no active FIB entry for a given set of prefixes, also produce a significant share of loss sequences. From its detection to its resolution, the recovery of a FIB entry may last several seconds after a link down operation (observation iii).

From this diagnostic, several improvements are possible for both router vendors and ISP. First, there already exists several solutions for dealing with (i) at the vendor side but they are rarely implemented or deployed. Dampening flappy interface at the up may be a feasible solution for solving (ii) at the manufacturer side, while (iii) may be mitigated both by using a better routing architecture enabling pre-provisioned backup paths and by a better calibration from the ISP of configurable updating timers. 
ISP tickets occur at another timescale. Their analysis provide more insight in the consequences of maintenance operations, and may help improve maintenance procedures. One could also envision automatic tickets pre-generation triggered by listener events and evolution of loss rates. Human ticket generation, which is often not very precise, could be assisted with data automatically retrieved from our monitoring architecture. In this topic of almost real time actions, the set of monitored flows could be adapted to locate faulty links. While monitoring all routes between all pairs of routers would be quite expensive in a large network, monitoring a set of pairs whose flows cover a maximum of links would be much more lightweight. In this case, adding some additional flows on demand would allow to get more information during a long lasting incident.

We envision that in a large network, probers could be attached to most routers, each prober generating only a small number of flows. When the loss rate on a flow grows above a predefined threshold, without any correlated routing event, additional flows could be launched to locate where losses occur, in the manner of tomography tools [29]. This could also allow to distinguish a DDoS, where we expect losses to occur along a tree towards the target from a single faulty link.

\section{Acknowledgments}

We are very grateful to RENATER for authorizing such an experiment on their network. Generally speaking, their feedback, support and assistance were very valuable for our work. We also want to thank Andreas Guillot, who worked with us on this project during his internship. He provided a precious help on parsing the listener data.

\section{Appendix A. Listener Events vs. Tickets}

As mentioned in Section 2.5, the ISP tickets are one of the four data sources provided by DCART. In Section 4.2, we highlighted their weakness to provide reliable and precise information, so we excluded them from multiple source correlations analyzed in Section 5 . In this appendix, we briefly discuss results obtained from correlation between listener events and tickets. These results are useful to understand the limits of tickets towards a fully automated analysis and can give hints to network operators in order to improve their ticketing system.

While listener events, loss sequences and loops have hopefully a short duration and a highly measured accuracy, NOC tickets have a much coarser time granularity and accuracy. Since, for the most part, they are manually written, times are often rounded up to the minute or even to the hour. Ticket times (some last for days or even weeks) often include whole maintenance windows or observation periods after an incident, which may last longer than the real network events. In other cases, individual events may not be detected by the NOC until a significant network outage appears. In order to correlate tickets with listener events, we decide to focus on long enough flapping events with a different aggregation model than the one used in Section 4.2. Indeed, NOC tickets reference logical links between two routers rather than directed IP links, independently of the direction or the number of parallel links involved. For enabling correlations between these two data sources, we introduce the notion of macro-flap. For a burst of raw events to be classified as such, we require that more than 14 of them occur for the same logical link in a given $24 \mathrm{~h}$ period or less. ${ }^{11}$ We aggregate LSP raw events on an undirected logical link basis (including so all parallel links in both directions between adjacent routers) per $24 \mathrm{~h}$ time window.

As expected, macro-flap events last longer than flap ones: $1 \mathrm{~h} 09$ on average for the former whereas it is $11 \mathrm{~h} 06$ for the latter. Although flaps and macro-flaps share a large proportion of raw events, such a distinction offers us the ability to manipulate two slightly different kinds of meta events. Considering the macro-flap model, busy days for isolated events falls to $66 \%$ instead of $74 \%$ for flaps. The median of the number of raw events in a macro-flap rises to 52 when considering only busy days related to macro-flap instead of 12 on the overall period. The average number of such meta events seems reasonable regarding the number of tickets: 1.07 macro-flaps compared to 1.8 tickets. However, if we define isolated events as the complementary set of macro-flaps events, their relative share increase to more than $10 \%$ instead of the $5 \%$ referring to classic flaps. Thus, when using the macro-flaps perspective, we have an average of about 4 isolated outages per day plus 1 macro-flap per day compared to less than 2 tickets per day only. In a significant number of cases, the NOC does not report all observed routing outages in tickets. We do not expect the NOC to report short isolated outages but only long periods of flapping that may repeatedly and severely hamper the connectivity. It is also worth to recall that at least $25 \%$ of loss sequences are related to flaps.

A ticket is correlated with a macro-flap if its time interval matches the one of the macro-flap and if they are spatially consistent, i.e. they have at least one network component in common (e.g. a router hostname that describes the location). We classify the nature of their correlation according to one of the 6 types given in Fig. 17(a). For example, types 3 and 4 correspond to events for which early signs are not detected by the NOC until the outage gets worse and is announced in a ticket. Our macro-flap processing detects such early signs and aggregates them into one meta event. Generally, first losses start even before the flap period. Types 5 and 6 typically correspond either to an incident ticket start time manually set down to a round number, or to a maintenance ticket announcing a maintenance window starting before the actual operation. If the macro-flap lasts longer (type 5), this may be the case of an incident prematurely closed or an end time optimistically rounded down. Finally, types 1 and 2 should not happen in practice. In the first case, flaps begin just after the ticket is closed, which tells us that the problem begins after the NOC announced that this problem is solved, whereas in the second case, it would seem that the NOC did not provide the correct time interval in the ticket. Based on this classification, only 88 macro-flaps (over 379) have been correlated with 102 tickets (over 893), giving 108 correlated pairs. As a matter of fact, one macro-flap may be correlated with more than one ticket (for example a maintenance ticket followed by a subsequent incident ticket), and one ticket may gather distinct macro-flaps (which have not been aggregated according to our model). If the low ratio of correlated tickets against the total ticket number is due to a large number of tickets (e.g., electrical maintenance, optical test or isolated outages that do not disconnect the network, etc.) which do not affect the network connectivity to the same extent as macro-flaps generally do, the low ratio of macro-flaps resulting in tickets means that many network events are not reported. Either because no traffic outage has been noticed or because these events are not detected by the NOC. Fig. 17(b) shows the distribution of correlated pairs according to our classification. We distinguish incident and maintenance tickets. Fortunately, types 1 and 2 are nearly nonexistent as expected. Types 3 and 4 are the most frequent. It is interesting to note that incident tickets represent the majority for these types, indicating that early signs of the further outage are often undetected by the NOC. It requires to enable a fine-grained calibration for setting up more reactive alarms. Types 5 and 6 , for their part, correspond in majority to maintenance tickets. This reveals either that window maintenance times account for other events (pre-outage and/or post-outage works) or that overestimated times are not updated in the closing message. Finally, types 4 and 5 include some tickets closed although corresponding macro-flaps still continue, which may indicate either that

\footnotetext{
${ }^{11}$ In practice, we performed several analysis to achieve this time-granularity trade-off. In particular, we retained the minimal values that almost optimize the

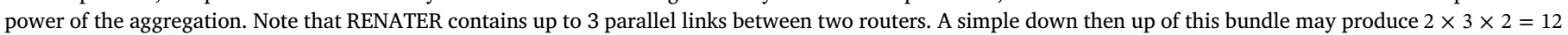
raw events.
} 


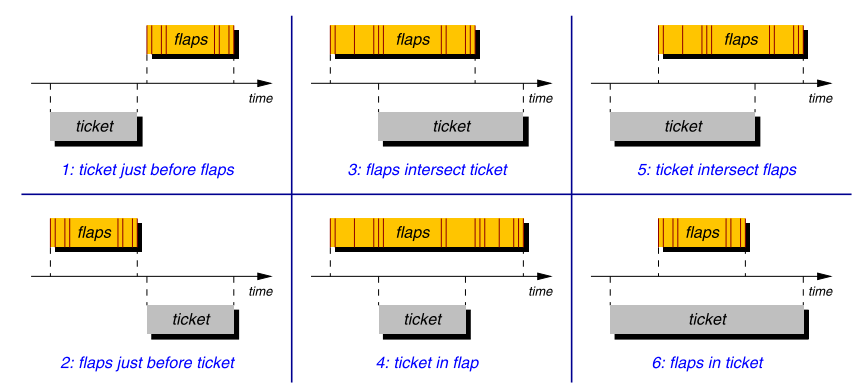

(a) Our temporal classification

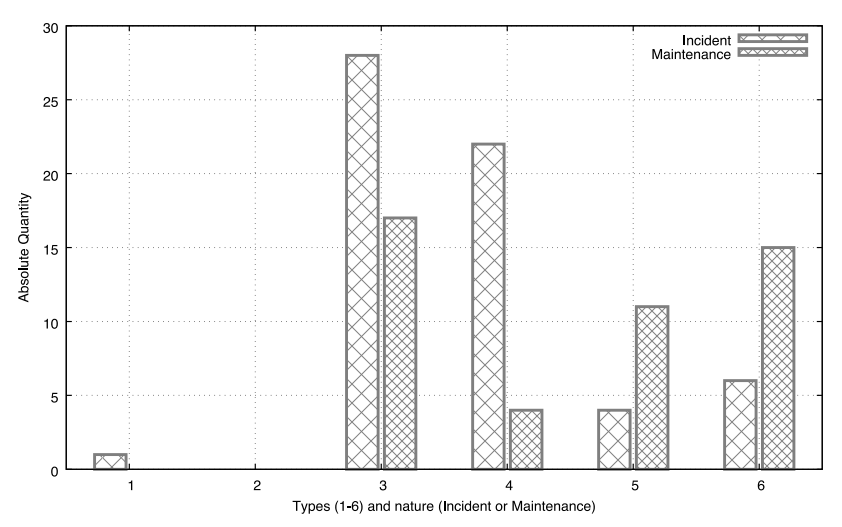

(b) Results distinguishing maintenance and incident tickets

Fig. 17. Flap/Ticket correlations.

tickets are optimistically closed (time rounded down) or problems are still not yet solved as advertised. No significant inconsistency has been discovered between tickets and flap events when they intersect. However, only a small share of flaps can be correlated with a NOC ticket, leaving many routing events, and their corresponding downtimes, unreported. Most of them do not imply a real connectivity loss and are not subject to a notification to network users. Correlated tickets often report problems which are detected sooner by the DCART listener. This suggests that DCART could help diagnosing problems before they provoke a noticeable downtime. Since lossy periods related to flaps account for $25 \%$ of loss sequences, we can reasonably conclude that a significant share of these losses are not covered by a ticket because they are under the radar of outages. Our analysis shows how our work could help an ISP to automatically pre-fill incident tickets to improve their accuracy and coverage.

\section{References}

[1] D. Oran, OSI is-IS Intra-domain routing protocol, RFC, 1142, IETF, 1990.

[2] P. Francois, Improving the convergence of ip routing protocols, Ph.d. thesis, (2007).

[3] C. Pelsser, L. Cittadini, S. Vissicchio, R. Bush, From paris to tokyo: on the suitability of ping to measure latency, Proceedings of the IMC, (2013).

[4] R. Mortier, PyRT: Python Routeing Toolkit, [Online]. Available: https://github. com/morl/pyrt.

[5] L. Blunk, M. Karir, C. Labovitz, MRT routing information export format, (RFC 6396), (2011).

[6] R. Ravaioli, G. Urvoy-Keller, C. Barakat, Characterizing ICMP rate limitation on routers, Proceedings of the ICC, (2015).

[7] F. Clad, Disruption-free routing convergence : computing minimal link-state update sequences, Université de Strasbourg, 2014 Ph.d. thesis. [Online]. Available: https:// tel.archives-ouvertes.fr/tel-01272168

[8] B. Donnet, M. Luckie, P. Mérindol, J.-J. Pansiot, Revealing MPLS tunnels obscured from traceroute, ACM SIGCOMM CCR 42 (2) (2012) 87.

[9] P. Francois, C. Filsfils, J. Evans, O. Bonaventure, Achieving sub-second IGP convergence in large IP networks, SIGCOMM Comput. Commun. Rev. 35 (3) (2005).

[10] E. Galstad, et al. Nagios, [Online]. Available: http://www.nagios.org.

[11] IBM, Tivoli NetView, [Online]. Available: http://www-03.ibm.com/software/ products/en/tivoli-netview-zos.

[12] S. Vissicchio, L. Cittadini, M. Pizzonia, L. Vergantini, V. Mezzapesa, M.L. Papagni, Beyond the best: real-time non-invasive collection of BGP messages, Proceedings of the internet network management workshop, san jose, ca, usa, (2010).

[13] A. Shaikh, A.G. Greenberg, OSPF monitoring - architecture, design, and deployment experience. Proceedings of the NSDI, (2004).

[14] G. Iannaccone, C.-N. Chuah, R. Mortier, S. Bhattacharyya, C. Diot, Analysis of link failures in an IP backbone, Proceedings of the 2nd ACM SIGCOMM Workshop on Internet Measurement, Marseille, France, (2002), pp. 237-242.

[15] A. Markopoulou, G. Iannaccone, S. Bhattacharyya, C.-N. Chuah, Y. Ganjali, C. Diot, Characterization of failures in an operational ip backbone network, IEEE/ACM Trans. Netw. 16 (4) (2008).

[16] A. Medem, M.-I. Akodjenou, R. Teixeira, TroubleMiner: mining network trouble tickets, Proceedings of the Integrated Network Management-Workshops, 2009. IM
'09, IEEE, New York, NY, 2009, pp. 113-119.

[17] A. Medem, R. Teixeira, N. Feamster, M. Meulle, Joint analysis ofNetwork incidents and intradomain routing changes, Proceedings of the CNSM, (2010).

[18] F. Wang, Z.M. Mao, J. Wang, L. Gao, R. Bush, A measurement study on the impact of routing events on end-to-end internet path performance, ACM SIGCOMM CCR 36 (4) (2006) 375.

[19] N. Feamster, D.G. Andersen, H. Balakrishnan, M.F. Kaashoek, Measuring the effects of internet path faults on reactive routing, ACM SIGMETRICS Perfor. Evaluat. Rev. 31 (1) (2003) 126.

[20] E.S. Myakotnykh, O.J. Wittner, B.E. Helvik, A. Abdelkefi, J.K. Hellan, O. Kvittem, T. Skjesol, A. Øslebø, An analysis of interdomain availability and causes of failures based on active measurements, Telecommun. Syst. (2011).

[21] P. Barford, J. Sommers, Comparing probe- and router-based packet-loss measurement. IEEE Int. Comput. (2004).

[22] A. Mahimkar, J. Yates, Y. Zhang, A. Shaikh, J. Wang, Z. Ge, C.T. Ee, Troubleshooting chronic conditions in large IP networks, the 2008 ACM CoNEXT Conference, ACM Press, New York, New York, USA, 2008, pp. 1-12.

[23] A. Mahimkar, H. Song, Z. Ge, A. Shaikh, J. Wang, J. Yates, Y. Zhang, J. Emmons, Detecting the performance impact of upgrades in large operational networks, ACM SIGCOMM CCR 40 (4) (2010) 303.

[24] A. Mahimkar, Z. Ge, A. Shaikh, J. Wang, J. Yates, Y. Zhang, Q. Zhao, Towards automated performance diagnosis in a large IPTV network, ACM SIGCOMM CCR 39 (4) (2009) 231.

[25] P. Francois, O. Bonaventure, Avoiding transient loops during the convergence of link-state routing protocols, IEEE/ACM Trans. Netw. 15 (6) (2007) 1280-1292.

[26] F. Clad, S. Vissicchio, P. Mérindol, P. Francois, J.J. Pansiot, Computing minimal update sequences for graceful router-wide reconfigurations, IEEE/ACM Trans. Netw. 23 (5) (2015) 1373-1386, https://doi.org/10.1109/TNET.2014.2332101.

[27] M. Shand, S. Bryant, A framework for loop-free convergence, RFC, 5715, IETF, 2010.

[28] U. Hengartner, S. Moon, R. Mortier, C. Diot, Detection and analysis of routing loops in packet traces, Proceedings of the 2nd ACM SIGCOMM Workshop on Internet Measurement, Marseille, France, (2002), pp. 107-112.

[29] L. Ma, T. He, A. Swami, D. Towsley, K.K. Leung, On optimal monitor placement for localizing node failures via network tomography, Perf. Eval. 91 (2015) 16-37. 